\title{
Interlayer Shifting in Two-Dimensional Covalent Organic Frameworks
}

Chengjun Kang, ${ }^{\dagger}$ Zhaoqiang Zhang, ${ }^{\dagger}$ Vanessa Wee ${ }^{\dagger}$ Adam K. Usadi, $₫$ David C. Calabro,$\ddagger$ Lisa Saunders Baugh, ${ }^{\ddagger}$ Shun Wang, ${ }^{\dagger}$ Yuxiang Wang, ${ }^{\dagger}$ Dan Zhao*†

†Department of Chemical and Biomolecular Engineering, National University of Singapore, 117585, Singapore

*Corporate Strategic Research Laboratory, ExxonMobil Research and Engineering Company, 1545 Route 22 East, Annandale, New Jersey 08801, United States

*E-mail: chezhao@nus.edu.sg 


\section{Experimental Details}

\section{Materials and Methods}

\subsection{Materials}

1,3,5-Tris(4-aminophenyl) benzene (> 93\%), terephthalaldehyde (>98\%), 1,4-dimethoxybenzene (> $97 \%)$, paraformaldehyde (> 90\%), hexamethylenetetramine (>99\%), 1,3,6,8-tetrabromopyrene (> 98\%), 4-(4,4,5,5,-tetramethyl-1,3,2-dioxaborolan-2-yl)aniline (> 98\%), 4-aminobenzonitrile (> 98\%), trifluoromethanesulfonic acid (> 98\%), 1,4-dioxane (> 99\%), tetrakis(triphenylphosphine)palladium(0) (> 97\%) were obtained from Tokyo Chemical Industry CO., LTD (Singapore). Other reagents and solvents were used as received.

\subsection{Methods}

Instruments and Methods. PXRD patterns were obtained on a Bruker D8 Advance X-ray powder diffractometer equipped with a Cu sealed tube $(\lambda=1.54178 \AA)$ at a scan rate of $3^{\circ} \mathrm{min}^{-1}$. Around 15 $\mathrm{mg}$ of COF powders were measured each time. As for the measurement of solvated COFs, $0.2-$ $0.5 \mathrm{~mL}$ of solvent was added to the dried sample (Note: the COF sample should be fully wetted without overflowing the solvent from the sample cell). Fourier-transform infrared spectroscopy (FTIR) was performed with a Bio-Rad FTS-3500 ARX FTIR spectrometer. Nuclear magnetic resonance spectroscopy (NMR) was conducted on a Bruker Avance $400 \mathrm{MHz}$ NMR spectrometer (DRX400) with chemical shifts being quoted in parts-per-million $(\mathrm{ppm})$ relative to tetramethylsilane. Fieldemission scanning electron microscopy (FESEM) images were taken on a JEOL JSM-7610F SEM. Samples were coated with Pt sputtering for $120 \mathrm{~s}$ before imaging.

Structural Modeling of COFs. Structural modeling of both dried and solvated TAPB-TA, TAPBOMeTA, Py-1P, and TAPT-BTCA COFs was carried out in the software package of Materials Studio (2017 R2(17.2.0.1626) version, Accelrys Software Inc.). P1 space group was used for all the quasi$A B$ stacking structures in the present study. In order to identify the model with predicted PXRD pattern that best match the experimental result, we used Powder Diffraction with "Mirror Diffraction" function. With this function, the predicted PXRD pattern can change automatically with the variation of model structures. Therefore, by continuously adjusting the model structure, we can compare the PXRD patterns of almost every possible interlayer-shifted COF structures with the experimental data, and finally identify the one particular structure that best match the experimental result. Theoretical models were initially optimized by the Forcite module, then Pawley refinements of the PXRD patterns were conducted in the Reflex module using 2 theta data from $1.5^{\circ}$ to $40^{\circ}$. The integrated intensities were extracted with the Pseudo-Voigt profile. As for the dried COFs, the unit cell parameters a, b, c, FWHM parameters, U, V, W, profile parameters NA, NB, and zero point were refined based on previous studies. ${ }^{1-4}$ As for the solvated COFs, in addition to the above parameters, alpha, beta, and gamma parameters were also refined. The background was refined with 20th order polynomial. The simulation data of the solvated COFs are provided in Tables S1-S4. 
DFT Calculation. First-principles density functional theory (DFT) calculations were performed in Castep software (Materials Studio, 2017 R2(17.2.0.1626) version, Accelrys Software Inc.). A semiempirical addition of dispersive forces to conventional DFT was included in the calculation to account for van der Waals interactions. Vanderbilt-type ultra-soft pseudopotentials and generalized gradient approximation (GGA) with a Perdew-Burke-Ernzerhof (PBE) exchange correlation and norm-conserving Troullier-Martins pseudopotentials in Kleinman-Bylander factorized form were used. In all calculations, we used periodic boundary conditions and a supercell large enough to present the full coordination environments of COFs. The Brillouin zone of the two-dimensional structures was sampled with 64 k-points (generated using the Monkhorst-Pack scheme), which ensures the convergence of the calculated total energies within $0.01 \mathrm{meV}^{\text {atom }}{ }^{-1}$. The atomic coordinates and unit-cell vectors were considered optimized when the remnant force on each atom was less than $0.04 \mathrm{eV} / \AA$. The structures of the simulated AA-stacked and quasi-AB-stacked COFs were firstly optimized, then solvent molecules were introduced to various locations of the channel pore, followed by a full structural relaxation to obtain a stable state of the material loaded with solvent molecules. To obtain the solvent binding energy, an isolated solvent molecule placed in a supercell (with the same cell dimensions as the material crystal) was also relaxed as a reference. 


\section{Synthesis of Building Blocks and COFs}

\subsection{Synthesis of 1,3,6,8-tetrakis(4-aminophenyl)pyrene ${ }^{4}$}

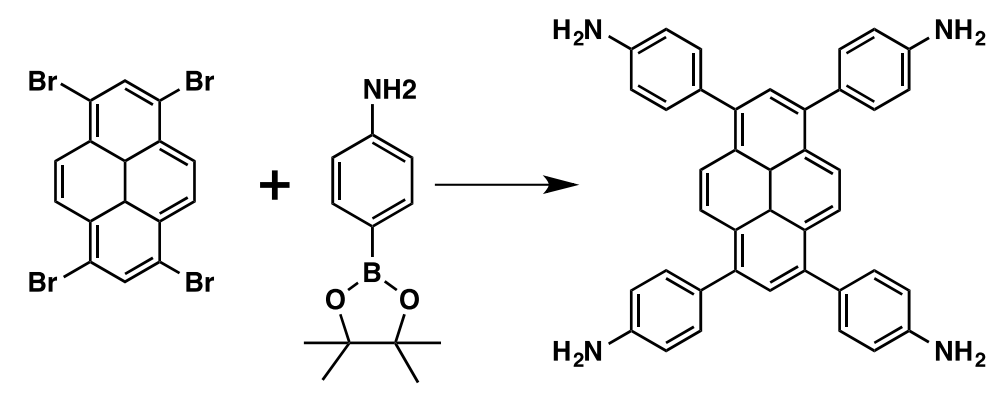

1,3,6,8-Tetrabromopyrene ( $0.74 \mathrm{~g}, 1.4 \mathrm{mmol}, 1.0$ eq.), 4-aminophenylboronic acid pinacol ester (1.5 g, $6.9 \mathrm{mmol}, 4.8$ eq.), $\mathrm{K}_{2} \mathrm{CO}_{3}\left(1.1 \mathrm{~g}, 7.9 \mathrm{mmol}, 5.5\right.$ eq.), and $\mathrm{Pd}\left(\mathrm{PPh}_{3}\right)_{4}$ (165 mg, $0.14 \mathrm{mmol}, 10$ $\mathrm{mol} \%$ ) were added to a flask under argon atmosphere. Then, degassed 1,4-dioxane (16 $\mathrm{mL})$ and $\mathrm{H}_{2} \mathrm{O}(4 \mathrm{~mL})$ were added and the mixture was refluxed $\left(115^{\circ} \mathrm{C}\right)$ for 3 days. After cooling to room temperature, a large excess of $\mathrm{H}_{2} \mathrm{O}$ was added to the mixture. The resulting light yellowish precipitate was collected via filtration and washed with $\mathrm{H}_{2} \mathrm{O}$ and $\mathrm{MeOH}$. The crude product was purified by dissolving in hot 1,4-dioxane and precipitating in water, filtered and dried under high vacuum. Bright yellow powder was obtained (yield: 81\%).

${ }^{1} \mathrm{H}$ NMR (400 MHz, DMSO-d6), ס: $8.13(4 \mathrm{H}), 7.79(2 \mathrm{H}), 7.34(8 \mathrm{H}), 6.77(8 \mathrm{H}), 5.30(8 \mathrm{H})$.

\subsection{Synthesis of 2,5-dimethoxyterephthalaldehyde ${ }^{5}$}<smiles>COc1ccc(OC)c(C=O)c1</smiles>

(a) Synthesis of 1,4-bis(chloromethyl)-2,5-dimethoxybenzene

1,4-Dioxane $(15 \mathrm{~mL})$ was added to a mixture of 1,4-dimethoxybenzene $(5.0 \mathrm{~g}, 36.2 \mathrm{mmol})$, formaldehyde solution $(2.5 \mathrm{~mL}, 37 \mathrm{wt} \%)$, and paraformaldehyde $(1.5 \mathrm{~g}, 50 \mathrm{mmol})$. The resultant mixture was heated to $90{ }^{\circ} \mathrm{C}$, and then concentrated $\mathrm{HCl}(5 \mathrm{~mL}, 37 \mathrm{wt} \%)$ was added dropwise over $5 \mathrm{~min}$. After the mixture was heated at $90{ }^{\circ} \mathrm{C}$ for another $1 \mathrm{~h}, \mathrm{HCl}(15 \mathrm{~mL}, 37 \mathrm{wt} \%)$ was added. Finally, the mixture was cooled to room temperature to afford white precipitate, which was collected by filtration and washed with water. The crude product was purified by recrystallization from acetone. A white powder product was obtained (yield: $85 \%$ ).

${ }^{1} \mathrm{H}$ NMR (400 MHz, $\left.\mathrm{CDCl}_{3}\right): \delta: 6.86(2 \mathrm{H}), 4.57(4 \mathrm{H}), 3.79(6 \mathrm{H})$.

(b) Synthesis of 2,5-dimethoxyterephthalaldehyde

Chloroform (25 mL) was added to a mixture of 1,4-bis(chloromethyl)-2,5-dimethoxybenzene (2.5 g, $10.6 \mathrm{mmol})$ and hexamethylenetetramine $(3.0 \mathrm{~g}, 21.3 \mathrm{mmol})$. The mixture was heated to $90{ }^{\circ} \mathrm{C}$ and stirred for $24 \mathrm{~h}$. Then, the mixture was cooled to room temperature to afford pale yellowish precipitate, which was collected by filtration, intensively washed with $\mathrm{CHCl}_{3}$, dried under vacuum, 
and re-dissolved in deionized water. Acetic acid $(5 \mathrm{~mL})$ was added to the aqueous mixture, which was heated to $90{ }^{\circ} \mathrm{C}$ and stirred for $24 \mathrm{~h}$. After cooling to room temperature, the mixture was extracted with dichloromethane (DCM) and dried by anhydrous $\mathrm{MgSO}_{4}$. $\mathrm{DCM}$ was removed by rotary evaporation, and the crude product was purified by recrystallization from ethanol. The purified product was obtained as a yellow solid (yield: $34 \%$ ).

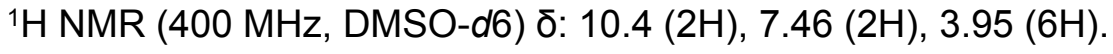

\subsection{Synthesis of 2,4,6-tris(4-aminophenyl)-1,3,5-triazine ${ }^{6}$}

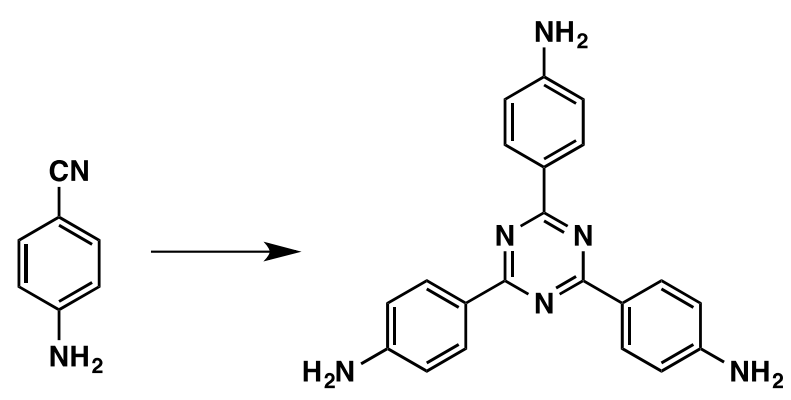

$\mathrm{CHCl}_{3}(5 \mathrm{~mL})$ was added to 4-aminobenzonitrile $(0.39 \mathrm{~g}, 3.2 \mathrm{mmol})$ in a round-bottomed flask. The flask was placed in an ice-water bath, and $\mathrm{CF}_{3} \mathrm{SO}_{3} \mathrm{H}(1.0 \mathrm{~mL}, 11.1 \mathrm{mmol})$ was added dropwise. The reaction mixture was stirred for $24 \mathrm{~h}$ at room temperature. Then, water $(10 \mathrm{~mL})$ was added, and the mixture was neutralized by aqueous $\mathrm{NaOH}$ solution $(2 \mathrm{M})$. The precipitate was filtered and dried under vacuum. Product was obtained as a pale yellow powder (yield: $90 \%$ ).

${ }^{1} \mathrm{H}$ NMR (400 MHz, DMSO) $\delta: ~ 8.36(6 \mathrm{H}), 6.70(6 \mathrm{H}), 5.92(6 \mathrm{H})$.

\subsection{Synthesis of TAPB-TA COF 1}

1,3,5-Tris(4-aminophenyl)benzene (55 $\mathrm{mg}, 0.16 \mathrm{mmol}$ ) and terephthalaldehyde (31 $\mathrm{mg}, 0.23 \mathrm{mmol})$ were dissolved in 1,4-dioxane $(5.0 \mathrm{~mL})$ with the aid of sonication. Then, mesitylene $(1.2 \mathrm{~mL})$ and distilled water $(1.2 \mathrm{~mL})$ were added to the mixture, and separate phases were observed. Glacial acetic acid $(1.8 \mathrm{~mL})$ was added to the mixture. The resultant homogeneous solution was sealed and heated at $65{ }^{\circ} \mathrm{C}$ for $24 \mathrm{~h}$. A yellow solid was isolated by filtration and washed with ethanol, immersed in ethanol, acetone, and tetrahydrofuran (THF) respectively for three days. After being dried under vacuum, the final COF was obtained as yellow powder (yield: $74 \%$ ).

\subsection{Synthesis of TAPB-OMeTA COF}

1,3,5-Tris(4-aminophenyl) benzene $(28.1 \mathrm{mg}, 0.080 \mathrm{mmol})$ and 2,5-dimethoxyterephthalaldehyde (19.9 $\mathrm{mg}, 0.120 \mathrm{mmol}$ ) were dispersed in a mixture of 1,2-dichlorobenzene $(0.5 \mathrm{~mL})$ and $n$-butanol $(0.5 \mathrm{~mL})$ inside a tube, then acetic acid $(0.1 \mathrm{~mL}, 6 \mathrm{M})$ was added to the mixture. The tube was sealed and heated at $120^{\circ} \mathrm{C}$ for three days. The precipitate was collected by filtration, washed with THF and ethanol, and immersed in THF and ethanol respectively for three days. After being dried under vacuum, the final COF was obtained as yellow powder (yield: $85 \%$ ).

\subsection{Synthesis of Py-1P COF}


1,3,6,8-Tetrakis(4-aminophenyl)pyrene $(14.0 \mathrm{mg}, 20 \mu \mathrm{mol})$ and terephthalaldehyde $(5.4 \mathrm{mg}, 40$ $\mu \mathrm{mol})$ were dispersed in a mixture of 1,4-dioxane $(0.3 \mathrm{~mL})$ and mesitylene $(0.7 \mathrm{~mL})$, then acetic acid $(0.1 \mathrm{~mL}, 6 \mathrm{M})$ was added to the mixture. The mixture was heated at $120^{\circ} \mathrm{C}$ for seven days. The precipitate was collected by filtration, washed with THF, immersed in THF and ethanol respectively for three days. After being dried under vacuum, the final COF was obtained as yellow powder (yield: $80 \%$ ).

\subsection{Synthesis of TAPT-BTCA COF²}

2,4,6-Tris(4-aminophenyl)-1,3,5-triazine (35.4 mg, $0.1 \mathrm{mmol}$ ) and 1,3,5-triformylbenzene (16.2 $\mathrm{mg}$, $0.1 \mathrm{mmol})$ were added to a mixture of 1,4-dioxane $(1.5 \mathrm{~mL})$ and mesitylene $(1.5 \mathrm{~mL})$. After a brief sonication, acetic acid was added $(0.2 \mathrm{~mL}, 6 \mathrm{M})$. The mixture was heated at $120{ }^{\circ} \mathrm{C}$ for three days. The precipitate was collected by filtration, washed with THF, immersed in THF and ethanol respectively for three days. After being dried under vacuum, the final COF was obtained as light yellow power (yield: 86\%). 
3. Additional Figures and Tables.

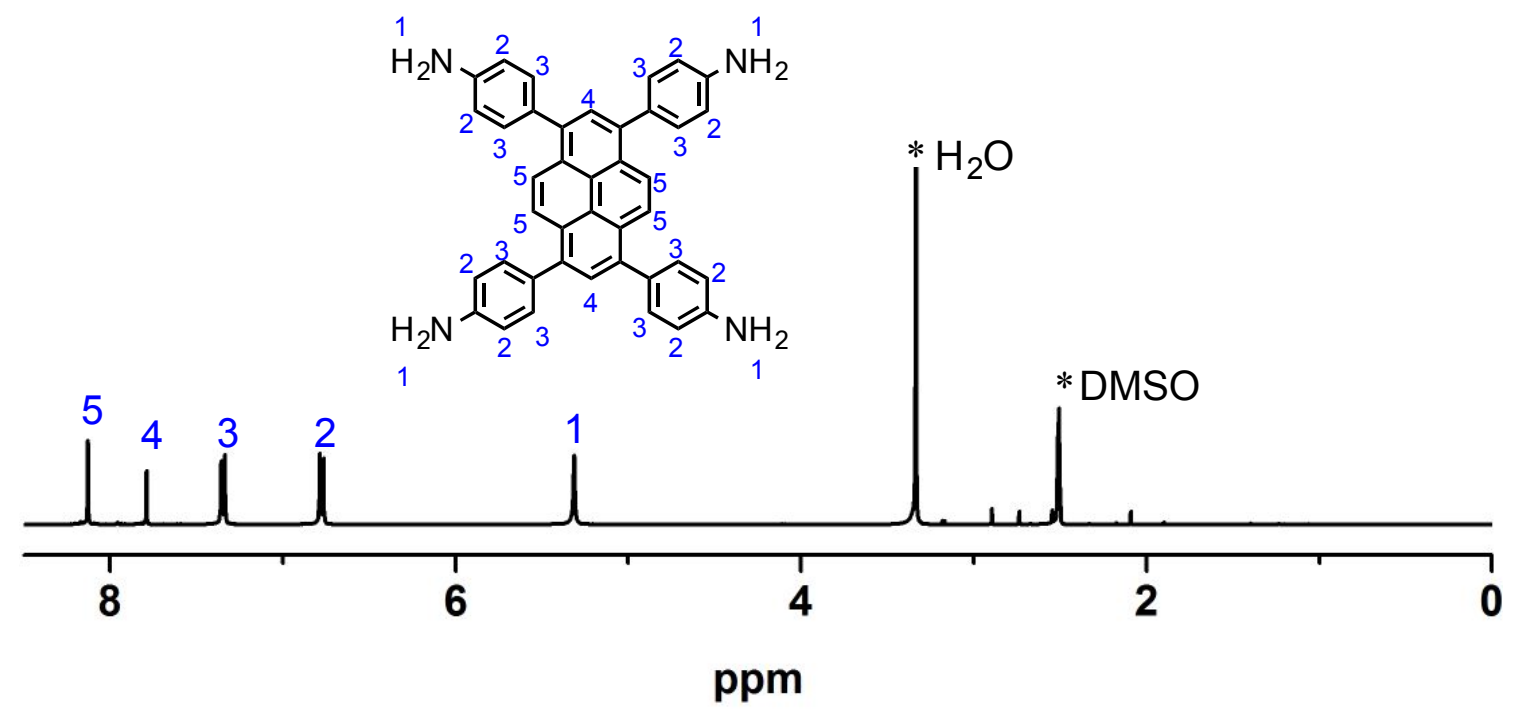

Figure S1. ${ }^{1} \mathrm{H}$ NMR spectrum of 1,3,6,8-tetrakis(4-aminophenyl)pyrene. 


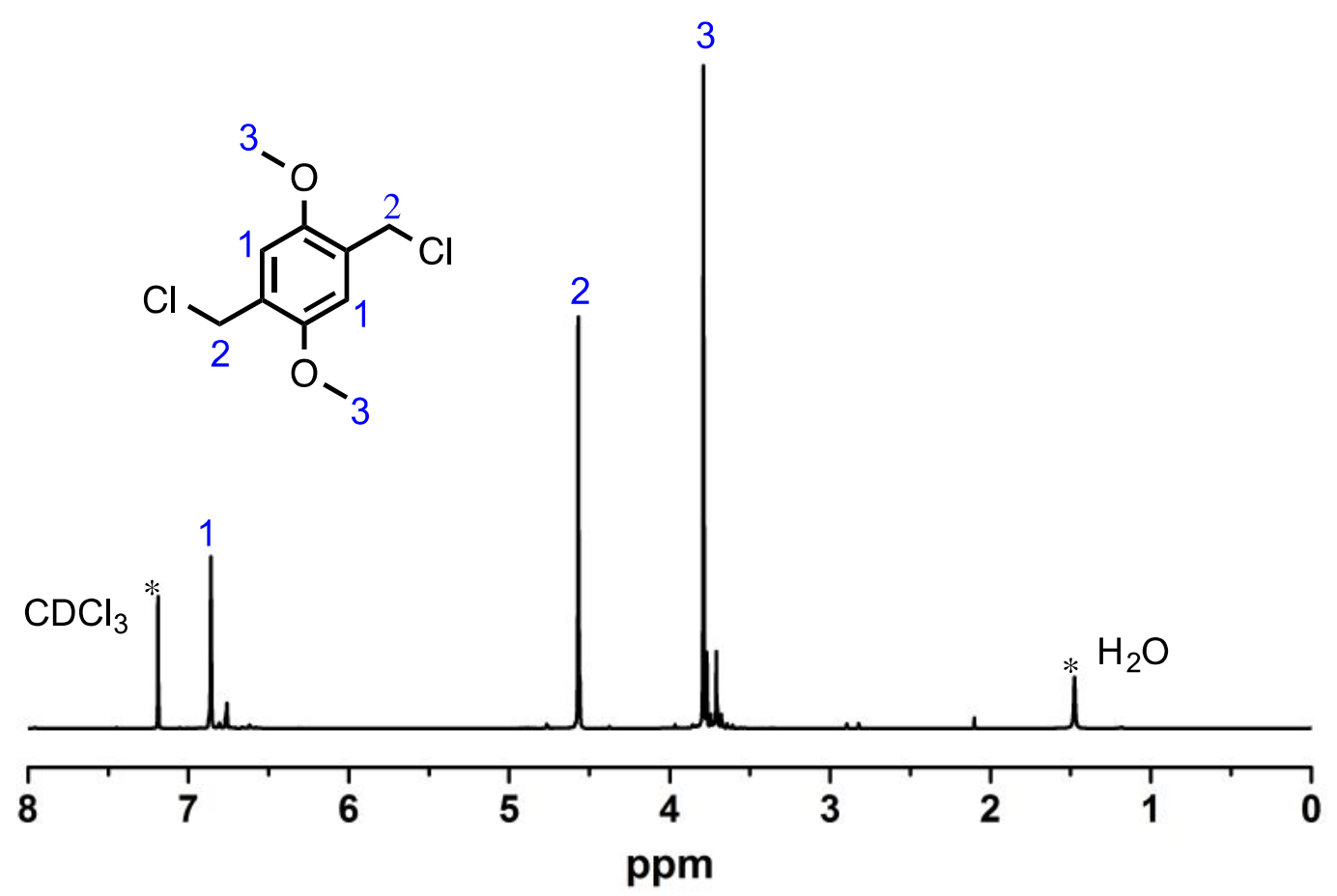

Figure S2. ${ }^{1} \mathrm{H}$ NMR spectrum of 1,4-bis(chloromethyl)-2,5-dimethoxybenzene. 


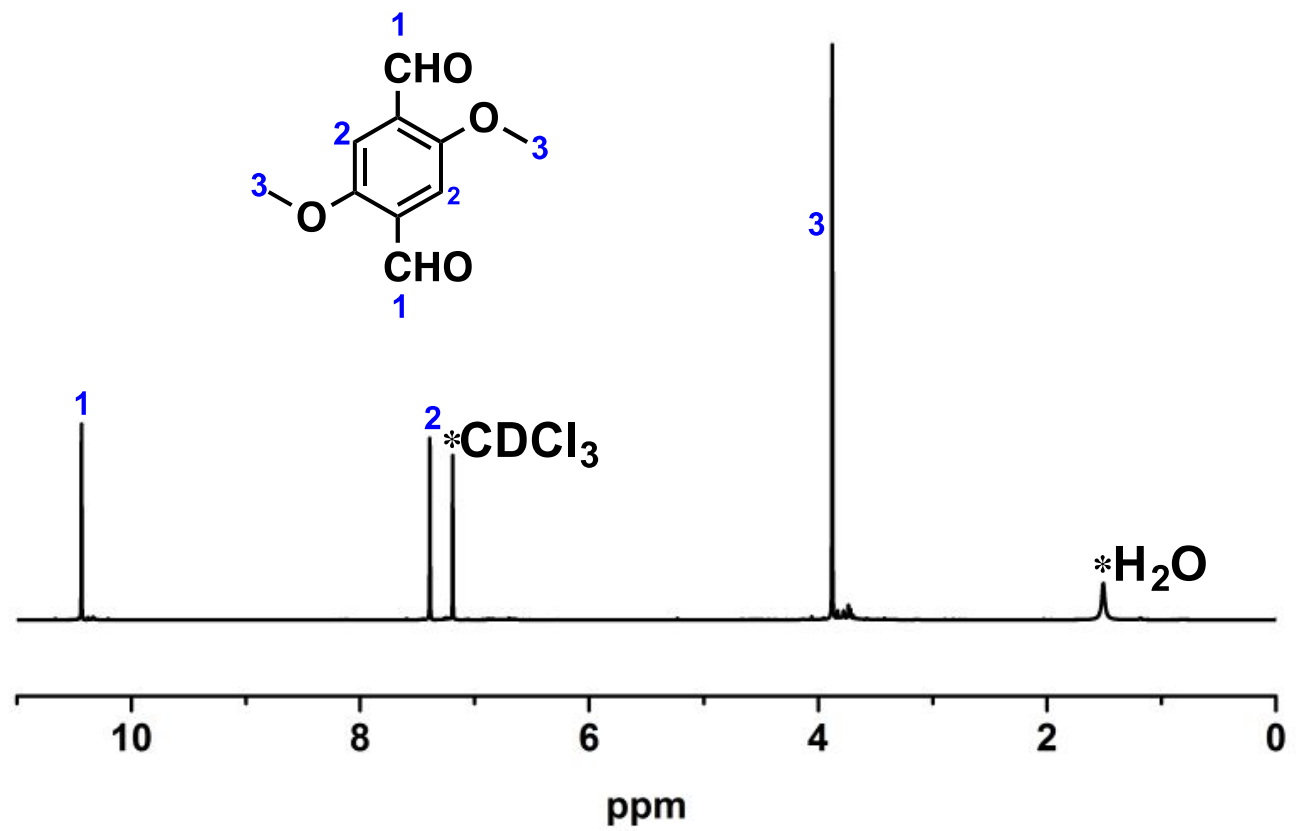

Figure S3. ${ }^{1} \mathrm{H}$ NMR spectrum of 2,5-dimethoxyterephthalaldehyde. 

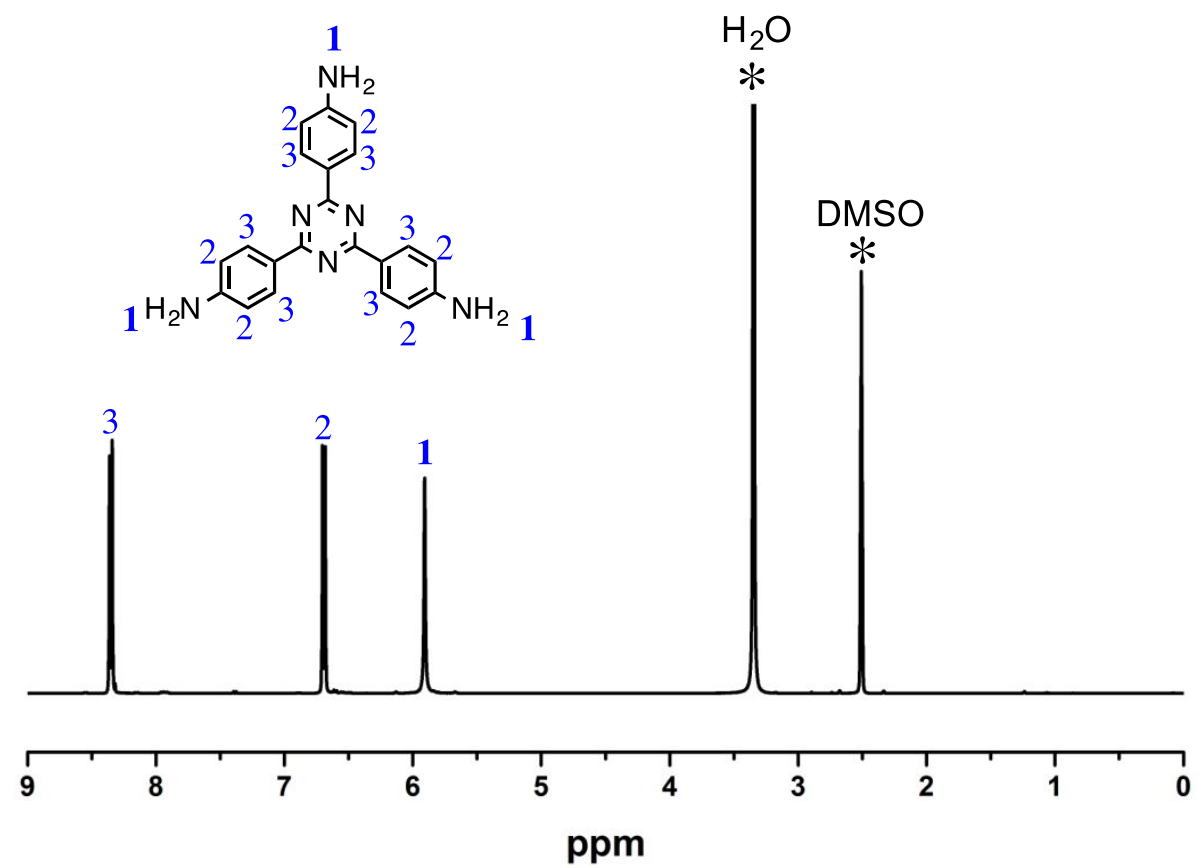

Figure S4. ${ }^{1} \mathrm{H}$ NMR spectrum of 2,4,6-tris(4-aminophenyl)-1,3,5-triazine. 


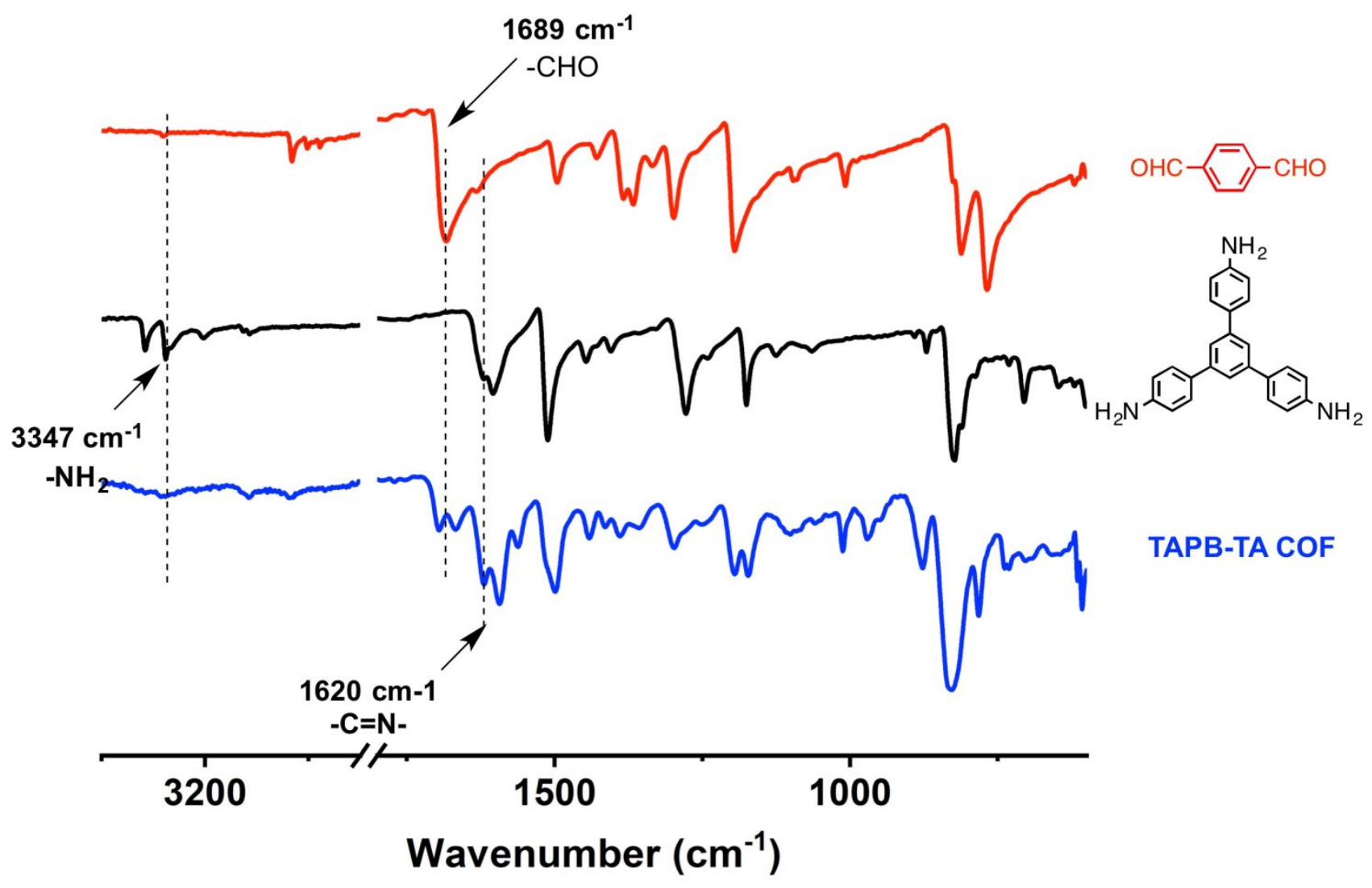

Figure S5. FTIR spectra of TAPB-TA COF and its monomers. 


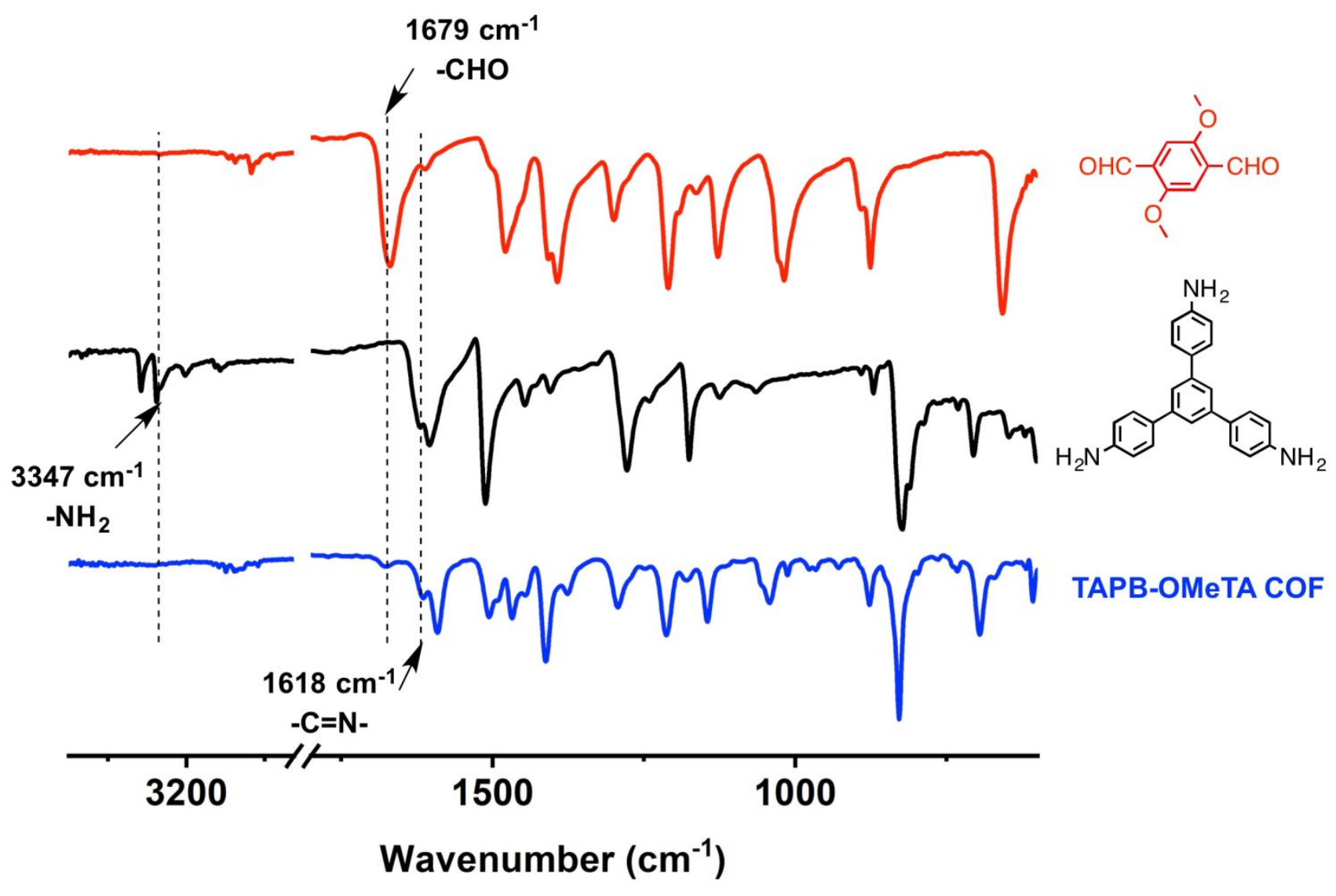

Figure S6. FTIR spectra of TAPB-OMeTA COF and its monomers. 


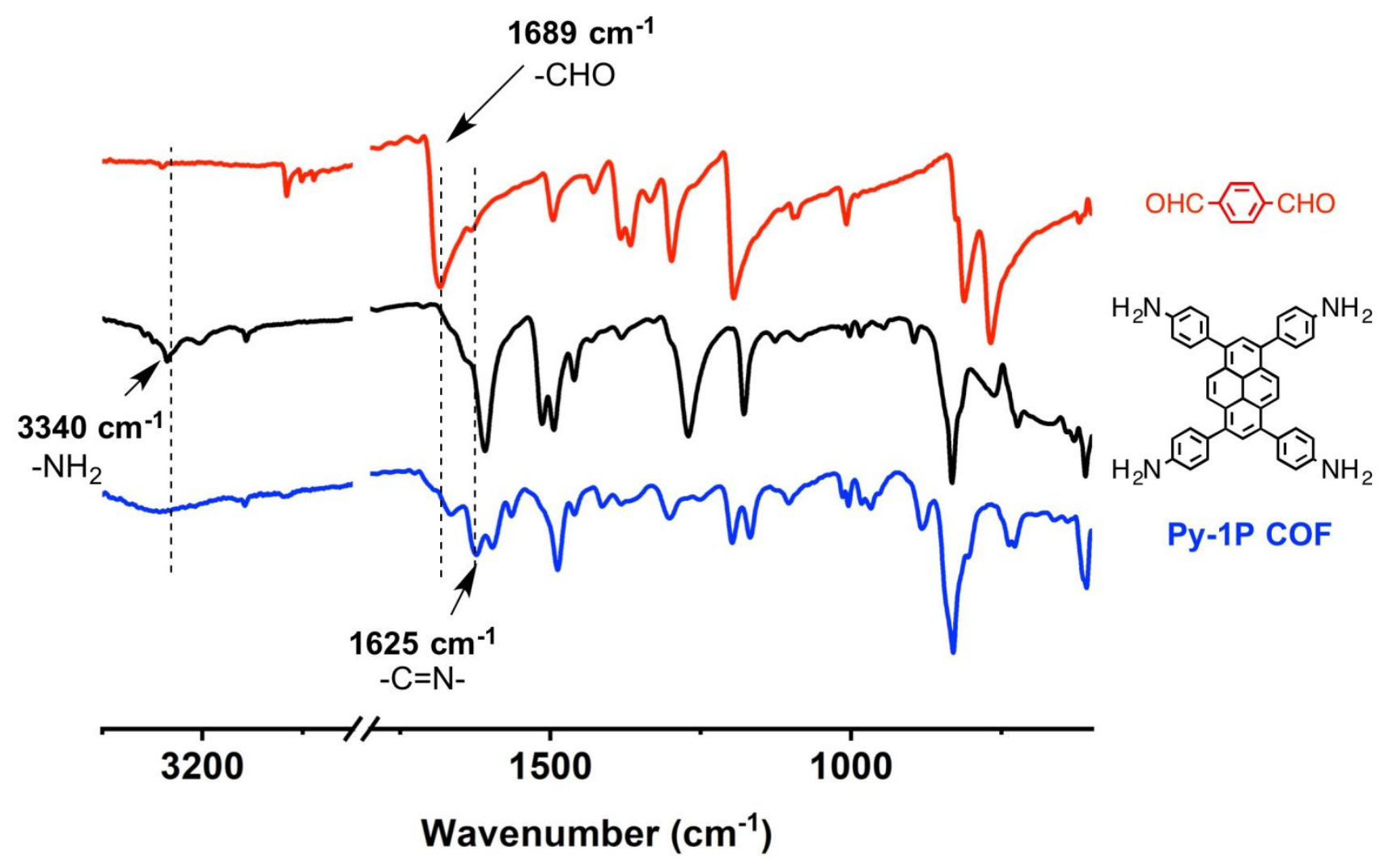

Figure S7. FTIR spectra of Py-1P COF and its monomers. 


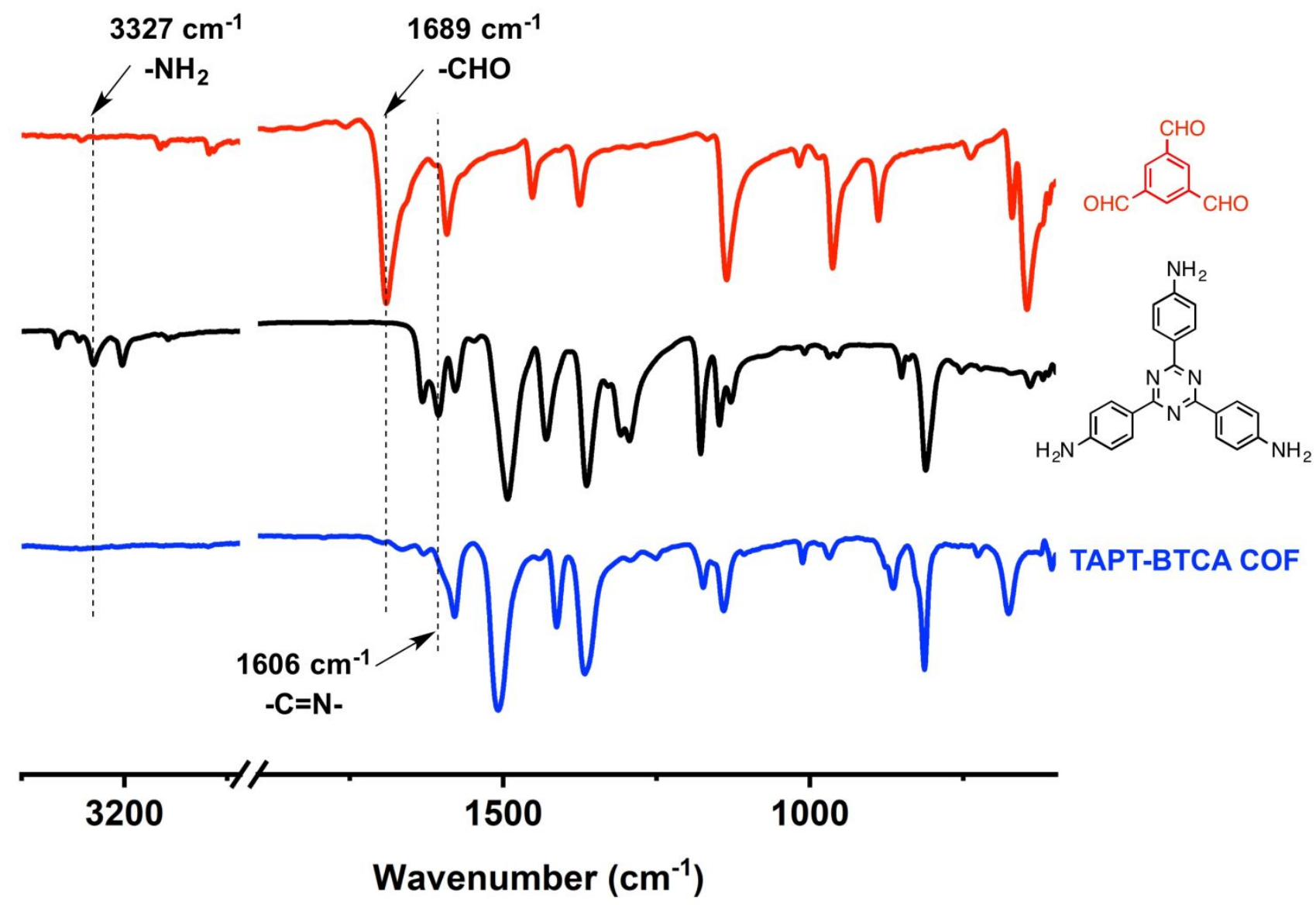

Figure S8. FTIR spectra of TAPT-BTCA COF and its monomers. 

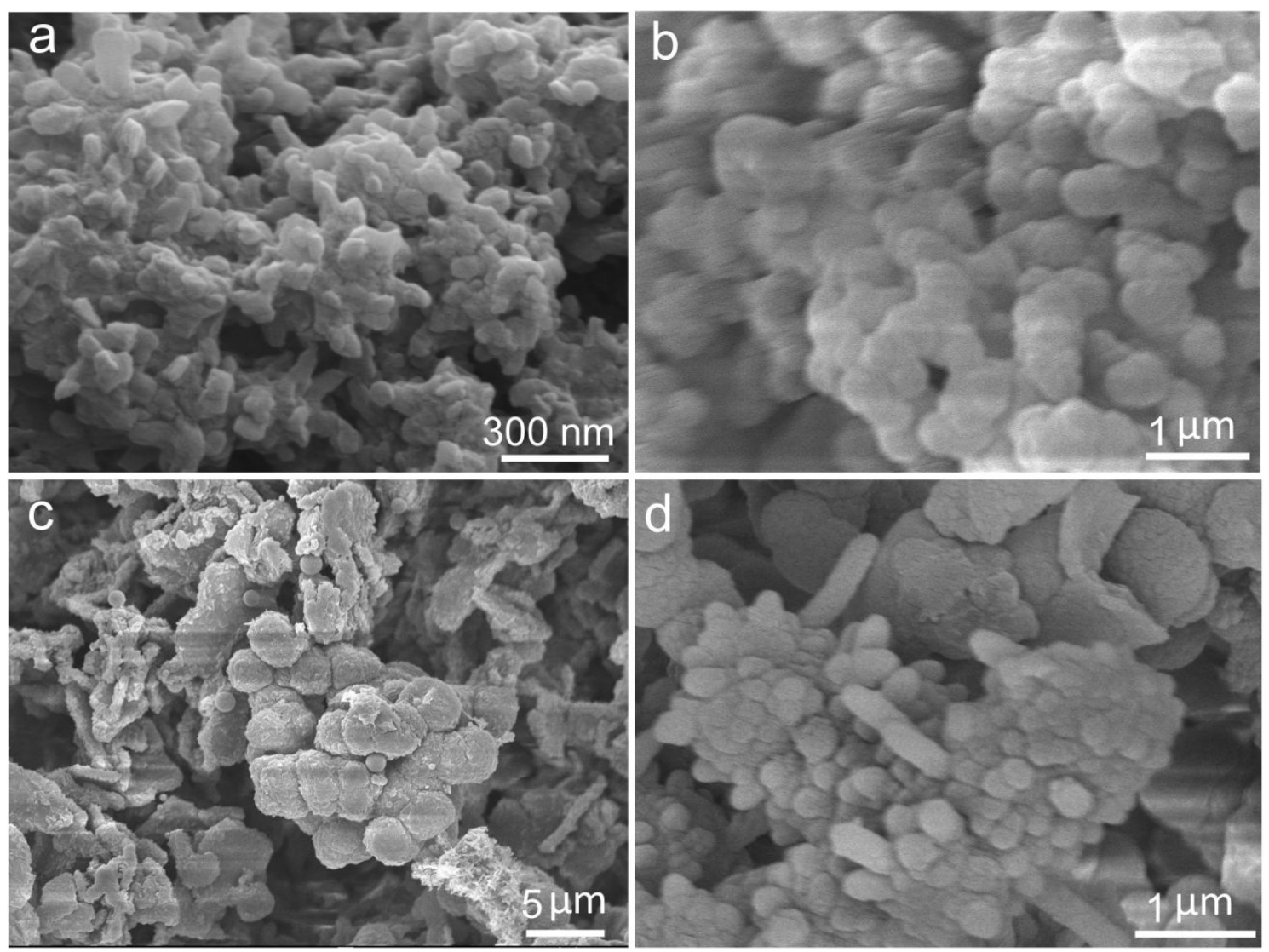

Figure S9. SEM images of the COFs in this study: (a) TAPB-TA COF, (b) TAPB-OMeTA COF, (c) Py-1P COF, (d) TAPT-BTCA COF. 


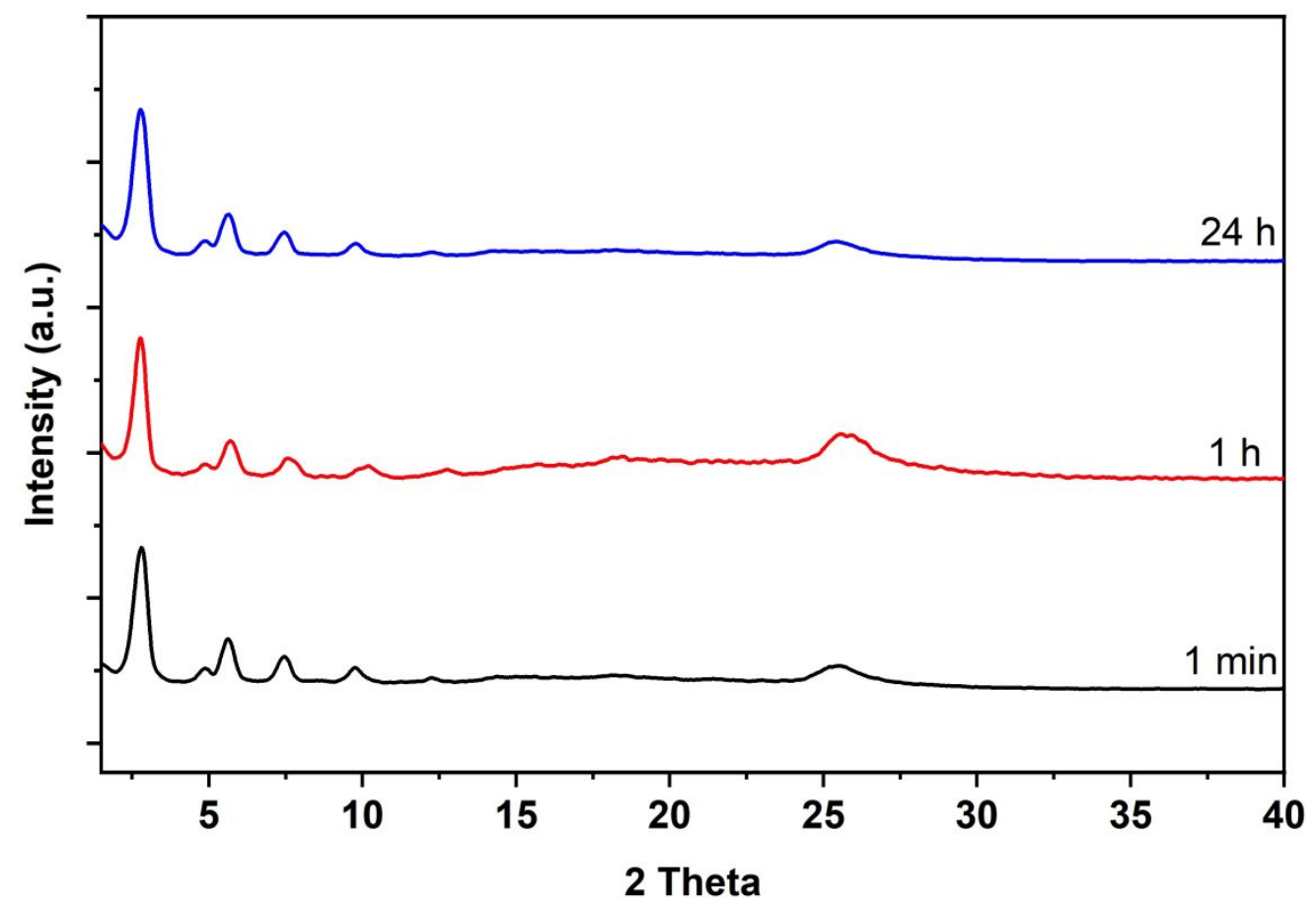

Figure S10. PXRD patterns of the TAPB-OMeTA COF with different soaking time in ethanol. 


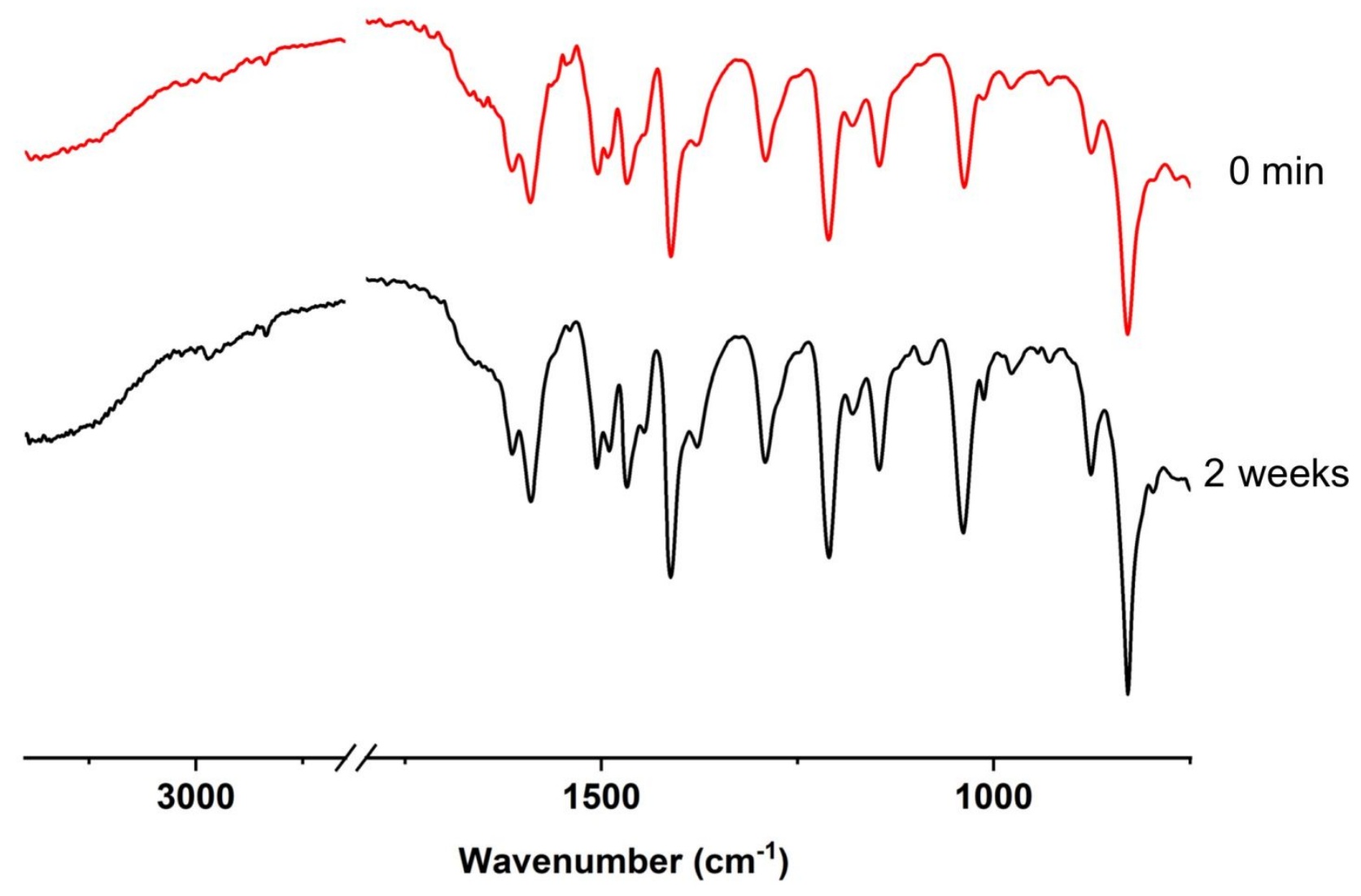

Figure S11. FTIR spectra of the TAPB-OMeTA COF with immersing time in ethanol for 0 min (red) and 2 weeks (black). 
Table S1. Detailed atom information of the simulated TAPB-TA COF in quasi-AB stacking mode.

$a=37.6143, b=37.4655, c=7.18442 ;$ alpha $=$ beta $=90^{\circ}$, gamma $=120^{\circ} ; P_{1}$ space group

\begin{tabular}{|c|c|c|c|c|}
\hline & & $x$ & $Y$ & $Z$ \\
\hline $\mathrm{C} 1$ & $C$ & 0.32363 & 0.79966 & 0.26725 \\
\hline $\mathrm{C} 2$ & $\mathrm{C}$ & 0.41433 & 0.61862 & 0.23629 \\
\hline C3 & C & 0.4573 & 0.64262 & 0.25031 \\
\hline $\mathrm{C} 4$ & C & 0.47726 & 0.68583 & 0.26352 \\
\hline $\mathrm{C} 5$ & $C$ & 0.45323 & 0.70498 & 0.26128 \\
\hline $\mathrm{C} 6$ & C & 0.41012 & 0.68198 & 0.24837 \\
\hline$C 7$ & C & 0.39125 & 0.63877 & 0.23653 \\
\hline $\mathrm{C} 8$ & C & 0.38494 & 0.70298 & 0.24734 \\
\hline C9 & $C$ & 0.39351 & 0.5726 & 0.22112 \\
\hline C10 & C & 0.52308 & 0.71093 & 0.27983 \\
\hline C11 & $C$ & 0.40031 & 0.74275 & 0.32395 \\
\hline C12 & $C$ & 0.37713 & 0.76248 & 0.32061 \\
\hline C13 & $C$ & 0.33739 & 0.74261 & 0.24442 \\
\hline C14 & $C$ & 0.32148 & 0.70287 & 0.17267 \\
\hline C15 & $\mathrm{C}$ & 0.34503 & 0.68356 & 0.17061 \\
\hline C16 & $C$ & 0.54577 & 0.69427 & 0.36095 \\
\hline C17 & $C$ & 0.58852 & 0.71755 & 0.37463 \\
\hline C18 & $C$ & 0.60974 & 0.75846 & 0.31009 \\
\hline C19 & $C$ & 0.58741 & 0.77545 & 0.23313 \\
\hline $\mathrm{C} 20$ & $C$ & 0.5448 & 0.75194 & 0.21503 \\
\hline C21 & $\mathrm{C}$ & 0.41381 & 0.5532 & 0.14187 \\
\hline $\mathrm{C} 22$ & $\mathrm{C}$ & 0.39436 & 0.51029 & 0.12895 \\
\hline $\mathrm{C} 23$ & $\mathrm{C}$ & 0.35383 & 0.48564 & 0.18865 \\
\hline $\mathrm{C} 24$ & $C$ & 0.33332 & 0.50456 & 0.26876 \\
\hline $\mathrm{C} 25$ & $\mathrm{C}$ & 0.35306 & 0.54739 & 0.28645 \\
\hline N26 & $\mathrm{N}$ & 0.3118 & 0.76099 & 0.24105 \\
\hline $\mathrm{H} 27$ & $\mathrm{H}$ & 0.47534 & 0.62755 & 0.25098 \\
\hline $\mathrm{H} 28$ & $\mathrm{H}$ & 0.46834 & 0.73804 & 0.27006 \\
\hline $\mathrm{H} 29$ & $\mathrm{H}$ & 0.35835 & 0.62053 & 0.22708 \\
\hline $\mathrm{H} 30$ & $\mathrm{H}$ & 0.42969 & 0.75854 & 0.3933 \\
\hline H31 & $\mathrm{H}$ & 0.39001 & 0.79251 & 0.38462 \\
\hline H32 & $\mathrm{H}$ & 0.29096 & 0.6872 & 0.11291 \\
\hline H33 & $\mathrm{H}$ & 0.3319 & 0.65389 & 0.10312 \\
\hline H34 & $\mathrm{H}$ & 0.53065 & 0.66365 & 0.42028 \\
\hline H35 & $\mathrm{H}$ & 0.60436 & 0.70372 & 0.44201 \\
\hline H36 & $\mathrm{H}$ & 0.6033 & 0.80689 & 0.1825 \\
\hline H37 & $\mathrm{H}$ & 0.52933 & 0.76593 & 0.14546 \\
\hline H38 & $\mathrm{H}$ & 0.44435 & 0.57092 & 0.0844 \\
\hline H39 & $\mathrm{H}$ & 0.41058 & 0.49618 & 0.06703 \\
\hline $\mathrm{H} 40$ & $\mathrm{H}$ & 0.30265 & 0.48649 & 0.32442 \\
\hline $\mathrm{H} 41$ & $\mathrm{H}$ & 0.33673 & 0.56036 & 0.35691 \\
\hline C42 & $\mathrm{C}$ & 0.29377 & 0.81426 & 0.26156 \\
\hline C43 & $\mathrm{C}$ & 0.25177 & 0.78692 & 0.23061 \\
\hline C44 & $\mathrm{C}$ & 0.22406 & 0.80145 & 0.22597 \\
\hline C45 & $\mathrm{C}$ & 0.23775 & 0.84345 & 0.25197 \\
\hline C46 & $\mathrm{C}$ & 0.27975 & 0.8708 & 0.28291 \\
\hline C47 & $\mathrm{C}$ & 0.30747 & 0.85626 & 0.28772 \\
\hline C48 & $\mathrm{C}$ & 0.20784 & 0.85798 & 0.24636 \\
\hline N49 & $\mathrm{N}$ & 0.21975 & 0.89681 & 0.26749 \\
\hline $\mathrm{H} 50$ & $\mathrm{H}$ & 0.35563 & 0.82205 & 0.28994 \\
\hline H51 & $\mathrm{H}$ & 0.2403 & 0.75434 & 0.20974 \\
\hline H52 & $\mathrm{H}$ & 0.19185 & 0.77982 & 0.2016 \\
\hline H53 & $\mathrm{H}$ & 0.29121 & 0.90337 & 0.30384 \\
\hline H54 & $\mathrm{H}$ & 0.33967 & 0.87787 & 0.31217 \\
\hline H55 & $\mathrm{H}$ & 0.1758 & 0.83541 & 0.22713 \\
\hline C56 & C & 0.19417 & 0.91523 & 0.26395 \\
\hline C57 & $\mathrm{C}$ & 0.15318 & 0.89412 & 0.20158 \\
\hline C58 & $\mathrm{C}$ & 0.12989 & 0.91373 & 0.20041 \\
\hline C59 & C & 0.14689 & 0.95473 & 0.26138 \\
\hline C60 & C & 0.18793 & 0.97575 & 0.32204 \\
\hline C61 & C & 0.21127 & 0.9562 & 0.32201 \\
\hline C62 & $\mathrm{C}$ & 0.12196 & 0.97546 & 0.26105 \\
\hline C63 & C & 0.08004 & 0.95331 & 0.30529 \\
\hline C64 & C & 0.05568 & 0.97203 & 0.29868 \\
\hline C65 & $\mathrm{C}$ & 0.07478 & 1.0145 & 0.25999 \\
\hline C66 & C & 0.11711 & 1.03774 & 0.22181 \\
\hline C67 & $\mathrm{C}$ & 0.13997 & 1.01741 & 0.21637 \\
\hline C68 & C & 0.01031 & 0.94688 & 0.32649 \\
\hline C69 & $\mathrm{C}$ & 0.13783 & 1.08329 & 0.19305 \\
\hline $\mathrm{C} 70$ & $\mathrm{C}$ & -0.01123 & 0.9052 & 0.27162 \\
\hline C71 & $\mathrm{C}$ & -0.05379 & 0.88185 & 0.28995 \\
\hline $\mathrm{C} 72$ & $\mathrm{C}$ & -0.07615 & 0.89962 & 0.35817 \\
\hline $\mathrm{C} 73$ & $\mathrm{C}$ & -0.055 & 0.94102 & 0.41628 \\
\hline
\end{tabular}




\begin{tabular}{|c|c|c|c|c|}
\hline $\mathrm{C} 74$ & C & -0.01222 & 0.96429 & 0.40139 \\
\hline C75 & C & 0.11661 & 1.10206 & 0.1177 \\
\hline C76 & C & 0.13574 & 1.14494 & 0.10189 \\
\hline C77 & C & 0.17665 & 1.17 & 0.15614 \\
\hline $\mathrm{C} 78$ & C & 0.19814 & 1.15151 & 0.23017 \\
\hline C79 & C & 0.17889 & 1.10877 & 0.24917 \\
\hline H80 & $\mathrm{H}$ & 0.13907 & 0.86296 & 0.14947 \\
\hline H81 & $\mathrm{H}$ & 0.09879 & 0.89701 & 0.14764 \\
\hline H82 & $\mathrm{H}$ & 0.20184 & 1.00708 & 0.37304 \\
\hline $\mathrm{H} 83$ & $\mathrm{H}$ & 0.24275 & 0.97288 & 0.37018 \\
\hline H84 & $\mathrm{H}$ & 0.06649 & 0.92143 & 0.34758 \\
\hline H85 & $\mathrm{H}$ & 0.05664 & 1.02949 & 0.25962 \\
\hline H86 & $\mathrm{H}$ & 0.17186 & 1.03413 & 0.17457 \\
\hline H87 & $\mathrm{H}$ & 0.00448 & 0.89071 & 0.20844 \\
\hline H88 & $\mathrm{H}$ & -0.06966 & 0.85 & 0.24493 \\
\hline H89 & $\mathrm{H}$ & -0.07109 & 0.95534 & 0.47688 \\
\hline $\mathrm{H} 90$ & $\mathrm{H}$ & 0.00308 & 0.9957 & 0.45166 \\
\hline H91 & $\mathrm{H}$ & 0.08537 & 1.08379 & 0.06867 \\
\hline H92 & $\mathrm{H}$ & 0.11874 & 1.15872 & 0.04509 \\
\hline H93 & $\mathrm{H}$ & 0.22917 & 1.16988 & 0.28137 \\
\hline H94 & $\mathrm{H}$ & 0.19591 & 1.09597 & 0.31501 \\
\hline N95 & $\mathrm{N}$ & -0.12003 & 0.87437 & 0.36731 \\
\hline C96 & C & -0.14618 & 0.88731 & 0.37938 \\
\hline C97 & C & -0.19071 & 0.85767 & 0.38051 \\
\hline C98 & C & -0.20591 & 0.81498 & 0.39124 \\
\hline C99 & C & -0.24813 & 0.78735 & 0.38545 \\
\hline C100 & C & 0.72409 & 0.8018 & 0.368 \\
\hline C101 & C & -0.26075 & 0.84451 & 0.35973 \\
\hline C102 & C & -0.21851 & 0.87216 & 0.36642 \\
\hline C103 & C & 0.67971 & 0.7719 & 0.35677 \\
\hline $\mathrm{N} 104$ & $\mathrm{~N}$ & 0.65353 & 0.7842 & 0.32499 \\
\hline N105 & $\mathrm{N}$ & 0.33509 & 0.44171 & 0.16998 \\
\hline C106 & C & 0.29588 & 0.41558 & 0.17042 \\
\hline C107 & C & 0.28026 & 0.37088 & 0.15554 \\
\hline C108 & C & 0.30707 & 0.35506 & 0.14193 \\
\hline C109 & C & 0.29158 & 0.31255 & 0.13352 \\
\hline C110 & C & 0.24912 & 0.2851 & 0.13849 \\
\hline C111 & C & 0.22228 & 0.30092 & 0.14958 \\
\hline C112 & C & 0.23778 & 0.34345 & 0.15799 \\
\hline C113 & C & 0.23373 & 1.24034 & 0.13798 \\
\hline N114 & $\mathrm{N}$ & 0.1946 & 1.21397 & 0.14378 \\
\hline H115 & $\mathrm{H}$ & -0.13591 & 0.91993 & 0.37809 \\
\hline H116 & $\mathrm{H}$ & -0.18521 & 0.80287 & 0.40312 \\
\hline H117 & $\mathrm{H}$ & -0.2591 & 0.75459 & 0.39279 \\
\hline $\mathrm{H} 118$ & $\mathrm{H}$ & 0.71853 & 0.85657 & 0.34697 \\
\hline $\mathrm{H} 119$ & $\mathrm{H}$ & -0.20751 & 0.90492 & 0.35808 \\
\hline $\mathrm{H} 120$ & $\mathrm{H}$ & 0.66969 & 0.73946 & 0.36905 \\
\hline $\mathrm{H} 121$ & $\mathrm{H}$ & 0.27391 & 0.42614 & 0.17935 \\
\hline $\mathrm{H} 122$ & $\mathrm{H}$ & 0.34 & 0.37546 & 0.1394 \\
\hline $\mathrm{H} 123$ & $\mathrm{H}$ & 0.31285 & 0.30107 & 0.12504 \\
\hline $\mathrm{H} 124$ & $\mathrm{H}$ & 0.18937 & 0.28048 & 0.15388 \\
\hline H125 & $\mathrm{H}$ & 0.21653 & 0.35494 & 0.16834 \\
\hline $\mathrm{H} 126$ & $\mathrm{H}$ & 0.25586 & 1.22992 & 0.13327 \\
\hline C127 & C & 0.58568 & 0.53479 & 0.75895 \\
\hline C128 & C & 0.67638 & 0.35375 & 0.72799 \\
\hline C129 & C & 0.71935 & 0.37775 & 0.74201 \\
\hline C130 & C & 0.73931 & 0.42096 & 0.75522 \\
\hline C131 & C & 0.71528 & 0.44011 & 0.75298 \\
\hline C132 & C & 0.67217 & 0.41711 & 0.74007 \\
\hline C133 & C & 0.6533 & 0.3739 & 0.72823 \\
\hline C134 & C & 0.64699 & 0.43811 & 0.73904 \\
\hline C135 & C & 0.65556 & 0.30773 & 0.71282 \\
\hline C136 & C & 0.78513 & 0.44606 & 0.77153 \\
\hline C137 & C & 0.66236 & 0.47788 & 0.81565 \\
\hline C138 & C & 0.63918 & 0.49761 & 0.81231 \\
\hline C139 & C & 0.59944 & 0.47774 & 0.73613 \\
\hline C140 & C & 0.58353 & 0.438 & 0.66438 \\
\hline C141 & C & 0.60708 & 0.41869 & 0.66231 \\
\hline C142 & C & 0.80782 & 0.4294 & 0.85266 \\
\hline C143 & C & 0.85057 & 0.45268 & 0.86633 \\
\hline C144 & C & 0.87179 & 0.49359 & 0.80179 \\
\hline C145 & C & 0.84946 & 0.51058 & 0.72484 \\
\hline C146 & C & 0.80685 & 0.48707 & 0.70673 \\
\hline C147 & C & 0.67586 & 0.28833 & 0.63357 \\
\hline C148 & C & 0.65641 & 0.24542 & 0.62065 \\
\hline C149 & C & 0.61588 & 0.22077 & 0.68035 \\
\hline C150 & C & 0.59537 & 0.23969 & 0.76046 \\
\hline
\end{tabular}




\begin{tabular}{|c|c|c|c|c|}
\hline C151 & C & 0.61511 & 0.28252 & 0.77815 \\
\hline N152 & $\mathrm{N}$ & 0.57385 & 0.49612 & 0.73275 \\
\hline H153 & $\mathrm{H}$ & 0.73739 & 0.36268 & 0.74268 \\
\hline H154 & $\mathrm{H}$ & 0.73039 & 0.47317 & 0.76177 \\
\hline H155 & $\mathrm{H}$ & 0.6204 & 0.35566 & 0.71878 \\
\hline H156 & $\mathrm{H}$ & 0.69174 & 0.49367 & 0.885 \\
\hline H157 & $\mathrm{H}$ & 0.65206 & 0.52764 & 0.87632 \\
\hline H158 & $\mathrm{H}$ & 0.55301 & 0.42233 & 0.60462 \\
\hline H159 & $\mathrm{H}$ & 0.59395 & 0.38902 & 0.59482 \\
\hline H160 & $\mathrm{H}$ & 0.7927 & 0.39878 & 0.91198 \\
\hline H161 & $\mathrm{H}$ & 0.86641 & 0.43885 & 0.93371 \\
\hline H162 & $\mathrm{H}$ & 0.86535 & 0.54202 & 0.67421 \\
\hline H163 & $\mathrm{H}$ & 0.79138 & 0.50106 & 0.63716 \\
\hline H164 & $\mathrm{H}$ & 0.7064 & 0.30605 & 0.5761 \\
\hline H165 & $\mathrm{H}$ & 0.67263 & 0.23131 & 0.55873 \\
\hline H166 & $\mathrm{H}$ & 0.5647 & 0.22162 & 0.81612 \\
\hline H167 & $\mathrm{H}$ & 0.59878 & 0.29549 & 0.84861 \\
\hline C168 & C & 0.55582 & 0.54939 & 0.75326 \\
\hline C169 & C & 0.51382 & 0.52205 & 0.72231 \\
\hline C170 & C & 0.48611 & 0.53658 & 0.71767 \\
\hline C171 & C & 0.4998 & 0.57858 & 0.74367 \\
\hline C172 & C & 0.5418 & 0.60593 & 0.77461 \\
\hline C173 & C & 0.56952 & 0.59139 & 0.77942 \\
\hline C174 & C & 0.46989 & 0.59311 & 0.73806 \\
\hline N175 & $\mathrm{N}$ & 0.4818 & 0.63194 & 0.75919 \\
\hline H176 & $\mathrm{H}$ & 0.61768 & 0.55718 & 0.78164 \\
\hline H177 & $\mathrm{H}$ & 0.50235 & 0.48947 & 0.70144 \\
\hline H178 & $\mathrm{H}$ & 0.4539 & 0.51495 & 0.6933 \\
\hline H179 & $\mathrm{H}$ & 0.55326 & 0.6385 & 0.79554 \\
\hline H180 & $\mathrm{H}$ & 0.60172 & 0.613 & 0.80388 \\
\hline H181 & $\mathrm{H}$ & 0.43785 & 0.57054 & 0.71884 \\
\hline C182 & C & 0.45622 & 0.65036 & 0.75565 \\
\hline C183 & C & 0.41523 & 0.62925 & 0.69329 \\
\hline C184 & C & 0.39194 & 0.64886 & 0.69212 \\
\hline C185 & C & 0.40894 & 0.68986 & 0.75308 \\
\hline C186 & C & 0.44998 & 0.71088 & 0.81374 \\
\hline C187 & C & 0.47332 & 0.69133 & 0.81371 \\
\hline C188 & C & 0.38401 & 0.71059 & 0.75275 \\
\hline C189 & C & 0.34209 & 0.68844 & 0.79699 \\
\hline C190 & C & 0.31773 & 0.70716 & 0.79038 \\
\hline C191 & C & 0.33683 & 0.74963 & 0.75169 \\
\hline C192 & C & 0.37916 & 0.77287 & 0.71351 \\
\hline C193 & C & 0.40202 & 0.75254 & 0.70808 \\
\hline C194 & C & 0.27236 & 0.68201 & 0.81819 \\
\hline C195 & C & 0.39988 & 0.81842 & 0.68476 \\
\hline C196 & C & 0.25082 & 0.64033 & 0.76332 \\
\hline C197 & C & 0.20826 & 0.61698 & 0.78165 \\
\hline C198 & C & 0.1859 & 0.63475 & 0.84987 \\
\hline C199 & C & 0.20705 & 0.67615 & 0.90799 \\
\hline C200 & C & 0.24983 & 0.69942 & 0.89309 \\
\hline C201 & C & 0.37866 & 0.83719 & 0.6094 \\
\hline C202 & C & 0.39779 & 0.88007 & 0.59359 \\
\hline C203 & C & 0.4387 & 0.90513 & 0.64784 \\
\hline C204 & C & 0.46019 & 0.88664 & 0.72187 \\
\hline C205 & C & 0.44094 & 0.8439 & 0.74087 \\
\hline H2O6 & $\mathrm{H}$ & 0.40112 & 0.59809 & 0.64117 \\
\hline $\mathrm{H} 207$ & $\mathrm{H}$ & 0.36084 & 0.63214 & 0.63934 \\
\hline H2O8 & $\mathrm{H}$ & 0.46389 & 0.74221 & 0.86474 \\
\hline H2O9 & $\mathrm{H}$ & 0.5048 & 0.70801 & 0.86188 \\
\hline $\mathrm{H} 210$ & $\mathrm{H}$ & 0.32854 & 0.65656 & 0.83928 \\
\hline $\mathrm{H} 211$ & $\mathrm{H}$ & 0.31869 & 0.76462 & 0.75133 \\
\hline H212 & $\mathrm{H}$ & 0.43391 & 0.76926 & 0.66627 \\
\hline H213 & $\mathrm{H}$ & 0.26653 & 0.62584 & 0.70015 \\
\hline H214 & $\mathrm{H}$ & 0.19239 & 0.58513 & 0.73663 \\
\hline $\mathrm{H} 215$ & $\mathrm{H}$ & 0.19096 & 0.69047 & 0.96858 \\
\hline H216 & $\mathrm{H}$ & 0.26513 & 0.73083 & 0.94336 \\
\hline H217 & $\mathrm{H}$ & 0.34742 & 0.81892 & 0.56037 \\
\hline H218 & $\mathrm{H}$ & 0.38079 & 0.89385 & 0.5368 \\
\hline H219 & $\mathrm{H}$ & 0.49122 & 0.90501 & 0.77307 \\
\hline H22O & $\mathrm{H}$ & 0.45796 & 0.8311 & 0.80671 \\
\hline N221 & $N$ & 0.14202 & 0.6095 & 0.85901 \\
\hline C222 & C & 0.11587 & 0.62244 & 0.87108 \\
\hline C223 & C & 0.07134 & 0.5928 & 0.87221 \\
\hline C224 & C & 0.05614 & 0.55011 & 0.88294 \\
\hline C225 & C & 0.01392 & 0.52248 & 0.87716 \\
\hline C226 & C & 0.98614 & 0.53693 & 0.8597 \\
\hline C227 & C & 0.0013 & 0.57964 & 0.85143 \\
\hline
\end{tabular}




$\begin{array}{llrrr}\mathrm{C} 228 & \mathrm{C} & 0.04354 & 0.60729 & 0.85813 \\ \mathrm{C} 229 & \mathrm{C} & 0.94176 & 0.50703 & 0.84847 \\ \mathrm{~N} 230 & \mathrm{~N} & 0.91558 & 0.51933 & 0.81669 \\ \mathrm{~N} 231 & \mathrm{~N} & 0.59714 & 0.17684 & 0.66168 \\ \mathrm{C} 232 & \mathrm{C} & 0.55793 & 0.15071 & 0.66212 \\ \mathrm{C} 233 & \mathrm{C} & 0.54231 & 0.10601 & 0.64724 \\ \mathrm{C} 234 & \mathrm{C} & 0.56912 & 0.09019 & 0.63363 \\ \mathrm{C} 235 & \mathrm{C} & 0.55363 & 0.04768 & 0.62522 \\ \mathrm{C} 236 & \mathrm{C} & 0.51117 & 0.02023 & 0.63019 \\ \mathrm{C} 237 & \mathrm{C} & 0.48433 & 0.03605 & 0.64128 \\ \mathrm{C} 238 & \mathrm{C} & 0.49983 & 0.07858 & 0.6497 \\ \mathrm{C} 239 & \mathrm{C} & 0.49578 & 0.97547 & 0.62968 \\ \mathrm{~N} 240 & \mathrm{~N} & 0.45665 & 0.9491 & 0.63548 \\ \mathrm{H} 241 & \mathrm{H} & 0.12614 & 0.65506 & 0.86979 \\ \mathrm{H} 242 & \mathrm{H} & 0.07684 & 0.538 & 0.89482 \\ \mathrm{H} 243 & \mathrm{H} & 0.00295 & 0.8845 \\ \mathrm{H} 244 & \mathrm{H} & 0.98058 & 0.5912 & 0.83867 \\ \mathrm{H} 245 & \mathrm{H} & 0.05454 & 0.64005 & 0.84978 \\ \mathrm{H} 246 & \mathrm{H} & 0.93174 & 0.47459 & 0.86076 \\ \mathrm{H} 247 & \mathrm{H} & 0.53596 & 0.16127 & 0.67105 \\ \mathrm{H} 248 & \mathrm{H} & 0.60205 & 0.6311 \\ \mathrm{H} 249 & \mathrm{H} & 0.5749 & 0.0362 & 0.61674 \\ \mathrm{H} 250 & \mathrm{H} & 0.45142 & 0.01561 & 0.64558 \\ \mathrm{H} 251 & \mathrm{H} & 0.47858 & 0.09007 & 0.66004 \\ \mathrm{H} 252 & \mathrm{H} & 0.51791 & 0.96505 & 0.62497\end{array}$


Table S2. Detailed atom information of the simulated TAPB-OMeTA COF in quasi-AB stacking mode.

$\mathrm{a}=37.2718, \mathrm{~b}=37.2718, \mathrm{c}=7.0430 ; \mathrm{alpha}=$ beta $=90^{\circ}$, gamma $=120^{\circ} ; \mathrm{P}_{1}$ space group

\begin{tabular}{|c|c|c|c|c|}
\hline & & $x$ & $Y$ & $Z$ \\
\hline C1 & $\mathrm{C}$ & 0.55938 & 0.01483 & 0.28567 \\
\hline $\mathrm{C} 2$ & $\mathrm{C}$ & 0.64625 & -0.16806 & 0.26255 \\
\hline $\mathrm{C} 3$ & $\mathrm{C}$ & 0.6896 & -0.14412 & 0.24946 \\
\hline $\mathrm{C} 4$ & $\mathrm{C}$ & 0.70925 & -0.1022 & 0.19819 \\
\hline $\mathrm{C} 5$ & $\mathrm{C}$ & 0.68527 & -0.08329 & 0.17277 \\
\hline C6 & $C$ & 0.64233 & -0.10537 & 0.20296 \\
\hline $\mathrm{C7}$ & $\mathrm{C}$ & 0.62294 & -0.14803 & 0.24166 \\
\hline $\mathrm{C} 8$ & $\mathrm{C}$ & 0.61835 & -0.08331 & 0.20303 \\
\hline C9 & $C$ & 0.62566 & -0.21388 & 0.29129 \\
\hline C10 & $\mathrm{C}$ & 0.75476 & -0.07822 & 0.17056 \\
\hline C11 & $\mathrm{C}$ & 0.63675 & -0.04237 & 0.27092 \\
\hline C12 & $C$ & 0.61403 & -0.02207 & 0.28385 \\
\hline C13 & $\mathrm{C}$ & 0.57211 & -0.04237 & 0.22986 \\
\hline C14 & $\mathrm{C}$ & 0.5537 & -0.08298 & 0.16139 \\
\hline C15 & $\mathrm{C}$ & 0.57659 & -0.1032 & 0.14594 \\
\hline C16 & $\mathrm{C}$ & 0.77641 & -0.09721 & 0.10058 \\
\hline C17 & $\mathrm{C}$ & 0.81913 & -0.07442 & 0.07341 \\
\hline C18 & $\mathrm{C}$ & 0.84174 & -0.03178 & 0.11559 \\
\hline C19 & $\mathrm{C}$ & 0.8212 & -0.01162 & 0.18346 \\
\hline $\mathrm{C} 20$ & $\mathrm{C}$ & 0.77864 & -0.03338 & 0.21388 \\
\hline $\mathrm{C} 21$ & $\mathrm{C}$ & 0.64539 & -0.23587 & 0.232 \\
\hline $\mathrm{C} 22$ & $\mathrm{C}$ & 0.62569 & -0.27877 & 0.24923 \\
\hline $\mathrm{C} 23$ & $\mathrm{C}$ & 0.58539 & -0.30113 & 0.3195 \\
\hline $\mathrm{C} 24$ & $\mathrm{C}$ & 0.56545 & -0.27971 & 0.38192 \\
\hline $\mathrm{C} 25$ & $\mathrm{C}$ & 0.58555 & -0.23655 & 0.36946 \\
\hline N26 & $\mathrm{N}$ & 0.54655 & -0.02404 & 0.25425 \\
\hline $\mathrm{N} 27$ & $N$ & 0.56626 & -0.34534 & 0.32344 \\
\hline N28 & $\mathrm{N}$ & 0.88558 & -0.00731 & 0.08655 \\
\hline $\mathrm{H} 29$ & $\mathrm{H}$ & 0.70841 & -0.15791 & 0.28136 \\
\hline H30 & $\mathrm{H}$ & 0.69998 & -0.0513 & 0.12758 \\
\hline H31 & $\mathrm{H}$ & 0.58972 & -0.16539 & 0.25672 \\
\hline H32 & $\mathrm{H}$ & 0.66832 & -0.02646 & 0.32265 \\
\hline H33 & $\mathrm{H}$ & 0.62911 & 0.00866 & 0.34439 \\
\hline H34 & $\mathrm{H}$ & 0.52141 & -0.09901 & 0.11967 \\
\hline H35 & $\mathrm{H}$ & 0.56152 & -0.13428 & 0.08858 \\
\hline H36 & $\mathrm{H}$ & 0.76026 & -0.12961 & 0.06113 \\
\hline H37 & $\mathrm{H}$ & 0.83392 & -0.09044 & 0.014 \\
\hline H38 & $\mathrm{H}$ & 0.83858 & 0.02116 & 0.21583 \\
\hline H39 & $\mathrm{H}$ & 0.76377 & -0.01736 & 0.27342 \\
\hline $\mathrm{H} 40$ & $\mathrm{H}$ & 0.67545 & -0.22049 & 0.16452 \\
\hline $\mathrm{H} 41$ & $\mathrm{H}$ & 0.64132 & -0.29495 & 0.20033 \\
\hline $\mathrm{H} 42$ & $\mathrm{H}$ & 0.53487 & -0.29574 & 0.44399 \\
\hline $\mathrm{H} 43$ & $\mathrm{H}$ & 0.56963 & -0.22124 & 0.42469 \\
\hline C44 & $\mathrm{C}$ & 0.52982 & 0.02975 & 0.3154 \\
\hline C45 & $\mathrm{C}$ & 0.48706 & 0.00253 & 0.3431 \\
\hline C46 & $\mathrm{C}$ & 0.4608 & 0.01906 & 0.36855 \\
\hline C47 & $\mathrm{C}$ & 0.47609 & 0.06185 & 0.36137 \\
\hline C48 & $C$ & 0.51902 & 0.08913 & 0.33862 \\
\hline C49 & $\mathrm{C}$ & 0.54529 & 0.07259 & 0.31329 \\
\hline C50 & $\mathrm{C}$ & 0.44585 & 0.07639 & 0.37338 \\
\hline N51 & $\mathrm{N}$ & 0.45748 & 0.11548 & 0.36514 \\
\hline H52 & $\mathrm{H}$ & 0.59209 & 0.03742 & 0.28523 \\
\hline H53 & $\mathrm{H}$ & 0.42814 & -0.00162 & 0.39501 \\
\hline H54 & $\mathrm{H}$ & 0.57794 & 0.09324 & 0.2864 \\
\hline H55 & $\mathrm{H}$ & 0.4133 & 0.05343 & 0.38507 \\
\hline C56 & $\mathrm{C}$ & 0.43011 & 0.13198 & 0.35567 \\
\hline C57 & $\mathrm{C}$ & 0.38999 & 0.10906 & 0.28032 \\
\hline C58 & $\mathrm{C}$ & 0.36551 & 0.12728 & 0.2575 \\
\hline C59 & $\mathrm{C}$ & 0.38005 & 0.16868 & 0.31267 \\
\hline $\mathrm{C} 60$ & $C$ & 0.42009 & 0.19108 & 0.39222 \\
\hline C61 & $C$ & 0.44497 & 0.17306 & 0.41004 \\
\hline C62 & $\mathrm{C}$ & 0.35456 & 0.18869 & 0.27482 \\
\hline C63 & $\mathrm{C}$ & 0.31131 & 0.16446 & 0.25575 \\
\hline C64 & $\mathrm{C}$ & 0.28746 & 0.18191 & 0.19607 \\
\hline C65 & $\mathrm{C}$ & 0.30703 & 0.22481 & 0.16729 \\
\hline C66 & $\mathrm{C}$ & 0.34946 & 0.25026 & 0.20276 \\
\hline C67 & $\mathrm{C}$ & 0.37317 & 0.23182 & 0.25038 \\
\hline C68 & $C$ & 0.24221 & 0.15534 & 0.16305 \\
\hline C69 & $\mathrm{C}$ & 0.36844 & 0.29606 & 0.19947 \\
\hline $\mathrm{C} 70$ & $\mathrm{C}$ & 0.22563 & 0.11438 & 0.09729 \\
\hline C71 & $\mathrm{C}$ & 0.18316 & 0.08952 & 0.06668 \\
\hline
\end{tabular}




\begin{tabular}{|c|c|c|c|c|}
\hline $\mathrm{C} 72$ & C & 0.15658 & 0.10523 & 0.0963 \\
\hline C73 & C & 0.17275 & 0.14591 & 0.16378 \\
\hline C74 & C & 0.2151 & 0.1706 & 0.19769 \\
\hline C75 & C & 0.34556 & 0.31458 & 0.26134 \\
\hline C76 & C & 0.36411 & 0.35752 & 0.27441 \\
\hline C77 & C & 0.40599 & 0.38282 & 0.23087 \\
\hline C78 & C & 0.42871 & 0.36475 & 0.16164 \\
\hline C79 & C & 0.41 & 0.32178 & 0.14523 \\
\hline N80 & $\mathrm{N}$ & 0.42427 & 0.42649 & 0.2613 \\
\hline N81 & $\mathrm{N}$ & 0.11316 & 0.07844 & 0.06566 \\
\hline H82 & $\mathrm{H}$ & 0.37819 & 0.07785 & 0.22846 \\
\hline H83 & $\mathrm{H}$ & 0.33602 & 0.10916 & 0.18715 \\
\hline H84 & $\mathrm{H}$ & 0.43247 & 0.22243 & 0.44287 \\
\hline H85 & $\mathrm{H}$ & 0.4759 & 0.19107 & 0.46806 \\
\hline H86 & $\mathrm{H}$ & 0.29586 & 0.13187 & 0.28979 \\
\hline H87 & $\mathrm{H}$ & 0.28932 & 0.23855 & 0.11529 \\
\hline H88 & $\mathrm{H}$ & 0.4061 & 0.25136 & 0.26946 \\
\hline H89 & $\mathrm{H}$ & 0.24541 & 0.1016 & 0.06615 \\
\hline H9O & $\mathrm{H}$ & 0.17095 & 0.05808 & 0.0163 \\
\hline H91 & $\mathrm{H}$ & 0.15271 & 0.1583 & 0.19654 \\
\hline H92 & $\mathrm{H}$ & 0.22633 & 0.20128 & 0.25553 \\
\hline H93 & $\mathrm{H}$ & 0.31375 & 0.29595 & 0.30767 \\
\hline H94 & $\mathrm{H}$ & 0.34625 & 0.37112 & 0.32673 \\
\hline H95 & $\mathrm{H}$ & 0.46064 & 0.38355 & 0.11785 \\
\hline H96 & $\mathrm{H}$ & 0.42813 & 0.30881 & 0.08952 \\
\hline C97 & C & 0.08701 & 0.09062 & 0.02492 \\
\hline H98 & $\mathrm{H}$ & 0.09766 & 0.12322 & 0.00414 \\
\hline C99 & C & 0.91091 & -0.02094 & 0.0537 \\
\hline H100 & $\mathrm{H}$ & 0.90015 & -0.05377 & 0.05319 \\
\hline C101 & C & 0.51215 & -0.4169 & 0.34403 \\
\hline C102 & C & 0.52743 & -0.37188 & 0.35132 \\
\hline H103 & $\mathrm{H}$ & 0.50525 & -0.36162 & 0.37297 \\
\hline O104 & 0 & 0.53444 & 0.13209 & 0.32648 \\
\hline C105 & C & 0.57315 & 0.15881 & 0.41744 \\
\hline 0106 & 0 & 0.47184 & -0.04034 & 0.35856 \\
\hline C107 & C & 0.43322 & -0.06742 & 0.26767 \\
\hline C108 & C & 0.46969 & 0.55594 & 0.37131 \\
\hline C109 & C & 0.45276 & 0.51261 & 0.36392 \\
\hline C110 & C & 0.47901 & 0.49685 & 0.31502 \\
\hline C111 & C & 0.5215 & 0.52396 & 0.2913 \\
\hline C112 & C & 0.53867 & -0.43312 & 0.31117 \\
\hline C113 & C & 0.46342 & 0.45222 & 0.28703 \\
\hline C114 & C & 0.95571 & 0.00801 & 0.03093 \\
\hline C115 & C & 0.98238 & -0.00823 & 0.03862 \\
\hline C116 & C & 0.02554 & 0.01763 & 0.02684 \\
\hline C117 & C & 0.04212 & 0.0609 & 0.01449 \\
\hline C118 & C & 0.01538 & 0.07694 & -0.00265 \\
\hline C119 & C & 0.97225 & 0.05108 & 0.00893 \\
\hline 0120 & 0 & 0.58135 & -0.4062 & 0.28339 \\
\hline C121 & C & 0.60903 & -0.41699 & 0.374 \\
\hline 0122 & 0 & 0.41071 & 0.48429 & 0.4053 \\
\hline C123 & C & 0.38563 & 0.49939 & 0.48636 \\
\hline 0124 & 0 & 0.05239 & 0.0014 & 0.04142 \\
\hline C125 & C & 0.04059 & -0.03694 & -0.05282 \\
\hline 0126 & 0 & 0.94536 & 0.0671 & -0.01248 \\
\hline C127 & C & 0.95631 & 0.10526 & 0.08161 \\
\hline H128 & $\mathrm{H}$ & 0.59741 & 0.17612 & 0.30977 \\
\hline H129 & $\mathrm{H}$ & 0.58365 & 0.142 & 0.51176 \\
\hline $\mathrm{H} 130$ & $\mathrm{H}$ & 0.56962 & 0.18166 & 0.50435 \\
\hline H131 & $\mathrm{H}$ & 0.43707 & -0.0901 & 0.181 \\
\hline H132 & $\mathrm{H}$ & 0.42241 & -0.0509 & 0.17312 \\
\hline H133 & $\mathrm{H}$ & 0.40903 & -0.0849 & 0.37539 \\
\hline H134 & $\mathrm{H}$ & 0.44999 & -0.43096 & 0.39716 \\
\hline H135 & $\mathrm{H}$ & 0.54138 & 0.51164 & 0.25263 \\
\hline H136 & $\mathrm{H}$ & 0.48551 & 0.44142 & 0.28762 \\
\hline H137 & $\mathrm{H}$ & 0.96949 & -0.04117 & 0.0608 \\
\hline H138 & $\mathrm{H}$ & 0.02831 & 0.10992 & -0.02376 \\
\hline H139 & $\mathrm{H}$ & 0.6264 & -0.42377 & 0.26599 \\
\hline $\mathrm{H} 140$ & $\mathrm{H}$ & 0.59307 & 0.55586 & 0.47003 \\
\hline H141 & $\mathrm{H}$ & 0.63175 & -0.39007 & 0.45867 \\
\hline H142 & $\mathrm{H}$ & 0.35755 & 0.47297 & 0.5491 \\
\hline H143 & $\mathrm{H}$ & 0.37548 & 0.51396 & 0.37847 \\
\hline H144 & $\mathrm{H}$ & 0.40262 & 0.52232 & 0.59866 \\
\hline H145 & $\mathrm{H}$ & 0.0673 & -0.03318 & -0.13783 \\
\hline H146 & $\mathrm{H}$ & 0.01379 & -0.04687 & -0.14984 \\
\hline H147 & $\mathrm{H}$ & 0.03302 & -0.06171 & 0.05293 \\
\hline H148 & $\mathrm{H}$ & 0.96375 & 0.12997 & -0.02424 \\
\hline
\end{tabular}




\begin{tabular}{|c|c|c|c|c|}
\hline H149 & $\mathrm{H}$ & 0.983 & 0.11561 & 0.17971 \\
\hline H150 & $\mathrm{H}$ & 0.92925 & 0.10101 & 0.1652 \\
\hline C151 & C & 0.55305 & 0.45411 & 0.83992 \\
\hline C152 & C & 0.63992 & 0.27121 & 0.8168 \\
\hline C153 & C & 0.68328 & 0.29515 & 0.80372 \\
\hline C154 & C & 0.70292 & 0.33708 & 0.75244 \\
\hline C155 & C & 0.67894 & 0.35598 & 0.72703 \\
\hline C156 & C & 0.636 & 0.3339 & 0.75721 \\
\hline C157 & C & 0.61661 & 0.29124 & 0.79592 \\
\hline C158 & C & 0.61202 & 0.35597 & 0.75728 \\
\hline C159 & C & 0.61934 & 0.2254 & 0.84555 \\
\hline C160 & C & 0.74843 & 0.36105 & 0.72481 \\
\hline C161 & C & 0.63043 & 0.3969 & 0.82518 \\
\hline C162 & C & 0.6077 & 0.4172 & 0.8381 \\
\hline C163 & C & 0.56578 & 0.3969 & 0.78411 \\
\hline C164 & C & 0.54737 & 0.35629 & 0.71565 \\
\hline C165 & C & 0.57026 & 0.33608 & 0.7002 \\
\hline C166 & C & 0.77008 & 0.34206 & 0.65483 \\
\hline C167 & C & 0.8128 & 0.36486 & 0.62766 \\
\hline C168 & C & 0.83542 & 0.40749 & 0.66984 \\
\hline C169 & C & 0.81487 & 0.42765 & 0.73772 \\
\hline C170 & C & 0.77231 & 0.4059 & 0.76813 \\
\hline C171 & C & 0.63906 & 0.20341 & 0.78626 \\
\hline C172 & C & 0.61936 & 0.1605 & 0.80348 \\
\hline C173 & C & 0.57906 & 0.13815 & 0.87375 \\
\hline C174 & C & 0.55912 & 0.15957 & 0.93617 \\
\hline C175 & C & 0.57922 & 0.20273 & 0.92371 \\
\hline N176 & $\mathrm{N}$ & 0.54022 & 0.41523 & 0.8085 \\
\hline N177 & $\mathrm{N}$ & 0.55993 & 0.09393 & 0.87769 \\
\hline N178 & $\mathrm{N}$ & 0.87925 & 0.43197 & 0.6408 \\
\hline H179 & $\mathrm{H}$ & 0.70208 & 0.28136 & 0.83562 \\
\hline H180 & $\mathrm{H}$ & 0.69366 & 0.38798 & 0.68184 \\
\hline H181 & $\mathrm{H}$ & 0.58339 & 0.27388 & 0.81098 \\
\hline H182 & $\mathrm{H}$ & 0.66199 & 0.41282 & 0.87691 \\
\hline H183 & $\mathrm{H}$ & 0.62278 & 0.44793 & 0.89865 \\
\hline H184 & $\mathrm{H}$ & 0.51508 & 0.34027 & 0.67392 \\
\hline H185 & $\mathrm{H}$ & 0.55519 & 0.30499 & 0.64283 \\
\hline H186 & $\mathrm{H}$ & 0.75394 & 0.30967 & 0.61538 \\
\hline H187 & $\mathrm{H}$ & 0.82759 & 0.34883 & 0.56825 \\
\hline H188 & $\mathrm{H}$ & 0.83225 & 0.46044 & 0.77008 \\
\hline H189 & $\mathrm{H}$ & 0.75744 & 0.42192 & 0.82767 \\
\hline H190 & $\mathrm{H}$ & 0.66912 & 0.21879 & 0.71877 \\
\hline H191 & $\mathrm{H}$ & 0.63499 & 0.14432 & 0.75459 \\
\hline H192 & $\mathrm{H}$ & 0.52854 & 0.14353 & 0.99824 \\
\hline H193 & $\mathrm{H}$ & 0.5633 & 0.21803 & 0.97894 \\
\hline C194 & C & 0.52349 & 0.46903 & 0.86965 \\
\hline C195 & C & 0.48074 & 0.44181 & 0.89735 \\
\hline C196 & C & 0.45447 & 0.45833 & 0.92281 \\
\hline C197 & C & 0.46976 & 0.50113 & 0.91562 \\
\hline C198 & C & 0.51269 & 0.52841 & 0.89288 \\
\hline C199 & $C$ & 0.53896 & 0.51187 & 0.86755 \\
\hline C200 & C & 0.43952 & 0.51566 & 0.92764 \\
\hline N201 & $\mathrm{N}$ & 0.45115 & 0.55475 & 0.91939 \\
\hline H2O2 & $\mathrm{H}$ & 0.58576 & 0.47669 & 0.83948 \\
\hline H2O3 & $\mathrm{H}$ & 0.42181 & 0.43765 & 0.94927 \\
\hline H2O4 & $\mathrm{H}$ & 0.57161 & 0.53252 & 0.84066 \\
\hline H205 & $\mathrm{H}$ & 0.40698 & 0.4927 & 0.93933 \\
\hline C206 & C & 0.42378 & 0.57125 & 0.90992 \\
\hline C207 & C & 0.38366 & 0.54834 & 0.83458 \\
\hline C208 & C & 0.35918 & 0.56656 & 0.81176 \\
\hline C209 & C & 0.37372 & 0.60795 & 0.86693 \\
\hline C210 & C & 0.41376 & 0.63035 & 0.94648 \\
\hline C211 & C & 0.43864 & 0.61233 & 0.96429 \\
\hline C212 & C & 0.34823 & 0.62796 & 0.82908 \\
\hline C213 & C & 0.30498 & 0.60374 & 0.81001 \\
\hline C214 & $C$ & 0.28113 & 0.62118 & 0.75033 \\
\hline C215 & C & 0.3007 & 0.66408 & 0.72154 \\
\hline C216 & C & 0.34313 & 0.68954 & 0.75702 \\
\hline C217 & C & 0.36684 & 0.6711 & 0.80464 \\
\hline C218 & C & 0.23588 & 0.59461 & 0.7173 \\
\hline C219 & C & 0.36211 & 0.73534 & 0.75372 \\
\hline C220 & C & 0.2193 & 0.55366 & 0.65154 \\
\hline C221 & C & 0.17683 & 0.52879 & 0.62093 \\
\hline C222 & C & 0.15025 & 0.5445 & 0.65056 \\
\hline C223 & C & 0.16642 & 0.58519 & 0.71804 \\
\hline C224 & C & 0.20877 & 0.60987 & 0.75194 \\
\hline C225 & C & 0.33923 & 0.75386 & 0.8156 \\
\hline
\end{tabular}




\begin{tabular}{|c|c|c|c|c|}
\hline C226 & C & 0.35778 & 0.7968 & 0.82866 \\
\hline C227 & C & 0.39966 & 0.82209 & 0.78513 \\
\hline C228 & C & 0.42238 & 0.80403 & 0.71589 \\
\hline C229 & C & 0.40367 & 0.76105 & 0.69949 \\
\hline N230 & $\mathrm{N}$ & 0.41794 & 0.86577 & 0.81556 \\
\hline N231 & $\mathrm{N}$ & 0.10683 & 0.51771 & 0.61991 \\
\hline $\mathrm{H} 232$ & $\mathrm{H}$ & 0.37186 & 0.51712 & 0.78271 \\
\hline H233 & $\mathrm{H}$ & 0.32969 & 0.54844 & 0.7414 \\
\hline $\mathrm{H} 234$ & $\mathrm{H}$ & 0.42614 & 0.6617 & 0.99713 \\
\hline H235 & $\mathrm{H}$ & 0.46957 & 0.63035 & 1.02231 \\
\hline H236 & $\mathrm{H}$ & 0.28953 & 0.57115 & 0.84404 \\
\hline H237 & $\mathrm{H}$ & 0.28299 & 0.67782 & 0.66955 \\
\hline H238 & $\mathrm{H}$ & 0.39978 & 0.69064 & 0.82371 \\
\hline H239 & $\mathrm{H}$ & 0.23908 & 0.54088 & 0.62041 \\
\hline $\mathrm{H} 240$ & $\mathrm{H}$ & 0.16462 & 0.49735 & 0.57055 \\
\hline H241 & $\mathrm{H}$ & 0.14638 & 0.59758 & 0.7508 \\
\hline $\mathrm{H} 242$ & $\mathrm{H}$ & 0.22 & 0.64056 & 0.80979 \\
\hline H243 & $\mathrm{H}$ & 0.30742 & 0.73523 & 0.86192 \\
\hline H244 & $\mathrm{H}$ & 0.33992 & 0.81039 & 0.88099 \\
\hline H245 & $\mathrm{H}$ & 0.45431 & 0.82282 & 0.6721 \\
\hline H246 & $\mathrm{H}$ & 0.4218 & 0.74809 & 0.64377 \\
\hline C247 & C & 0.08068 & 0.5299 & 0.57917 \\
\hline H248 & $\mathrm{H}$ & 0.09134 & 0.5625 & 0.5584 \\
\hline C249 & C & 0.90458 & 0.41833 & 0.60795 \\
\hline H250 & $\mathrm{H}$ & 0.89382 & 0.3855 & 0.60744 \\
\hline C251 & C & 0.50582 & 0.02238 & 0.89828 \\
\hline C252 & C & 0.5211 & 0.0674 & 0.90557 \\
\hline $\mathrm{H} 253$ & $\mathrm{H}$ & 0.49893 & 0.07766 & 0.92722 \\
\hline O254 & 0 & 0.52811 & 0.57137 & 0.88073 \\
\hline C255 & C & 0.56682 & 0.59809 & 0.97169 \\
\hline O256 & 0 & 0.46551 & 0.39894 & 0.91282 \\
\hline C257 & C & 0.42689 & 0.37186 & 0.82193 \\
\hline C258 & C & 0.46336 & 0.99522 & 0.92557 \\
\hline C259 & C & 0.44643 & 0.95188 & 0.91817 \\
\hline C260 & C & 0.47268 & 0.93613 & 0.86927 \\
\hline C261 & C & 0.51517 & 0.96323 & 0.84556 \\
\hline C262 & C & 0.53234 & 0.00616 & 0.86542 \\
\hline C263 & C & 0.4571 & 0.89149 & 0.84128 \\
\hline C264 & C & 0.94938 & 0.44729 & 0.58518 \\
\hline C265 & C & 0.97605 & 0.43104 & 0.59287 \\
\hline C266 & C & 0.01921 & 0.45691 & 0.58109 \\
\hline C267 & C & 0.03579 & 0.50017 & 0.56874 \\
\hline C268 & C & 0.00906 & 0.51622 & 0.55161 \\
\hline C269 & C & 0.96592 & 0.49036 & 0.56319 \\
\hline $\mathrm{O} 270$ & 0 & 0.57502 & 0.03307 & 0.83764 \\
\hline C271 & C & 0.6027 & 0.02229 & 0.92825 \\
\hline $\mathrm{O} 272$ & 0 & 0.40439 & 0.92356 & 0.95955 \\
\hline C273 & C & 0.37931 & 0.93867 & 1.04061 \\
\hline 0274 & 0 & 0.04606 & 0.44068 & 0.59568 \\
\hline C275 & C & 0.03426 & 0.40234 & 0.50143 \\
\hline 0276 & 0 & 0.93903 & 0.50637 & 0.54178 \\
\hline C277 & C & 0.94999 & 0.54453 & 0.63586 \\
\hline H278 & $\mathrm{H}$ & 0.59109 & 0.6154 & 0.86402 \\
\hline H279 & $\mathrm{H}$ & 0.57732 & 0.58127 & 1.06602 \\
\hline $\mathrm{H} 280$ & $\mathrm{H}$ & 0.56329 & 0.62094 & 1.0586 \\
\hline H281 & $\mathrm{H}$ & 0.43074 & 0.34917 & 0.73525 \\
\hline H282 & $\mathrm{H}$ & 0.41608 & 0.38838 & 0.72738 \\
\hline H283 & $\mathrm{H}$ & 0.4027 & 0.35438 & 0.92964 \\
\hline H284 & $\mathrm{H}$ & 0.44366 & 0.00832 & 0.95142 \\
\hline H285 & $\mathrm{H}$ & 0.53505 & 0.95092 & 0.80688 \\
\hline H286 & $\mathrm{H}$ & 0.47919 & 0.88069 & 0.84187 \\
\hline H287 & $\mathrm{H}$ & 0.96316 & 0.39811 & 0.61505 \\
\hline $\mathrm{H} 288$ & $\mathrm{H}$ & 0.02198 & 0.54919 & 0.53049 \\
\hline H289 & $\mathrm{H}$ & 0.62007 & 0.0155 & 0.82025 \\
\hline H290 & $\mathrm{H}$ & 0.58674 & 0.99514 & 1.02428 \\
\hline H291 & $\mathrm{H}$ & 0.62542 & 0.04921 & 1.01292 \\
\hline H292 & $\mathrm{H}$ & 0.35123 & 0.91224 & 1.10336 \\
\hline H293 & $\mathrm{H}$ & 0.36915 & 0.95324 & 0.93272 \\
\hline H294 & $\mathrm{H}$ & 0.39629 & 0.9616 & 1.15291 \\
\hline H295 & $\mathrm{H}$ & 0.06097 & 0.4061 & 0.41642 \\
\hline H296 & $\mathrm{H}$ & 0.00746 & 0.39241 & 0.40442 \\
\hline H297 & $\mathrm{H}$ & 0.0267 & 0.37757 & 0.60718 \\
\hline H298 & $\mathrm{H}$ & 0.95742 & 0.56925 & 0.53001 \\
\hline H299 & $\mathrm{H}$ & 0.97667 & 0.55489 & 0.73396 \\
\hline H300 & $\mathrm{H}$ & 0.92292 & 0.54029 & 0.71946 \\
\hline
\end{tabular}


Table S3. Detailed atom information of the simulated Py-1P COF in quasi-AB stacking mode. $\mathrm{a}=35.4000, \mathrm{~b}=33.9000, \mathrm{c}=8.7400 ;$ alpha $=90.0^{\circ}$, beta $=75.2^{\circ}$, gamma $=90.0^{\circ} ; \mathrm{P}_{1}$ space group

\begin{tabular}{|c|c|c|c|c|}
\hline & & $x$ & $Y$ & $Z$ \\
\hline $\mathrm{C} 1$ & $C$ & -0.20164 & 0.33753 & 0.68138 \\
\hline $\mathrm{C} 2$ & $C$ & -0.2374 & 0.33569 & 0.62352 \\
\hline C3 & C & -0.24975 & 0.3702 & 0.56267 \\
\hline $\mathrm{C} 4$ & $C$ & -0.22846 & 0.40515 & 0.55576 \\
\hline C5 & C & -0.25872 & 0.29804 & 0.62852 \\
\hline $\mathrm{H} 6$ & $\mathrm{H}$ & -0.19134 & 0.31163 & 0.73091 \\
\hline $\mathrm{H} 7$ & $\mathrm{H}$ & -0.27572 & 0.37022 & 0.51877 \\
\hline $\mathrm{C} 8$ & $\mathrm{C}$ & -0.27382 & 0.92566 & 0.45959 \\
\hline $\mathrm{C} 9$ & $C$ & -0.24048 & 0.89919 & 0.45917 \\
\hline $\mathrm{H} 10$ & $\mathrm{H}$ & -0.19641 & 0.94509 & 0.42199 \\
\hline $\mathrm{H} 11$ & $\mathrm{H}$ & -0.27618 & 0.84655 & 0.49922 \\
\hline $\mathrm{C} 12$ & C & 0.06214 & 0.69409 & 0.33901 \\
\hline C13 & C & 0.02167 & 0.69259 & 0.37037 \\
\hline C14 & $C$ & 0.00105 & 0.65821 & 0.43039 \\
\hline C15 & $\mathrm{C}$ & 0.0225 & 0.62328 & 0.44213 \\
\hline C16 & $\mathrm{C}$ & 0.06419 & 0.62468 & 0.40678 \\
\hline C17 & $C$ & 0.08389 & 0.66103 & 0.36256 \\
\hline C18 & $C$ & 0.08595 & 0.58997 & 0.4185 \\
\hline C19 & C & 0.06541 & 0.55448 & 0.4595 \\
\hline $\mathrm{C} 20$ & $C$ & 0.0251 & 0.55256 & 0.48151 \\
\hline $\mathrm{C} 21$ & C & 0.00253 & 0.58679 & 0.48361 \\
\hline $\mathrm{C} 22$ & C & -0.04035 & 0.65738 & 0.47485 \\
\hline $\mathrm{C} 23$ & C & -0.05922 & 0.62183 & 0.52752 \\
\hline $\mathrm{C} 24$ & $\mathrm{C}$ & -0.03916 & 0.58599 & 0.52338 \\
\hline $\mathrm{C} 25$ & $C$ & 0.12493 & 0.6632 & 0.33926 \\
\hline $\mathrm{C} 26$ & C & 0.14579 & 0.62871 & 0.34946 \\
\hline $\mathrm{C} 27$ & $C$ & 0.12742 & 0.59206 & 0.38914 \\
\hline $\mathrm{C} 28$ & $C$ & 0.14693 & 0.70123 & 0.32051 \\
\hline $\mathrm{C} 29$ & $C$ & 0.15327 & 0.55793 & 0.40485 \\
\hline C30 & C & -0.06585 & 0.69188 & 0.46253 \\
\hline C31 & $\mathrm{C}$ & -0.06399 & 0.54931 & 0.55041 \\
\hline C32 & $C$ & 0.15172 & 0.52144 & 0.32947 \\
\hline C33 & $C$ & 0.17803 & 0.49102 & 0.33845 \\
\hline C34 & $C$ & 0.20747 & 0.49709 & 0.41819 \\
\hline C35 & $C$ & 0.20996 & 0.5338 & 0.48762 \\
\hline C36 & $C$ & 0.18289 & 0.5635 & 0.48396 \\
\hline C37 & $C$ & 0.18248 & 0.70543 & 0.20461 \\
\hline C38 & $C$ & 0.20567 & 0.73911 & 0.2009 \\
\hline C39 & C & 0.19361 & 0.76928 & 0.31386 \\
\hline $\mathrm{C} 40$ & $C$ & 0.15763 & 0.76555 & 0.42574 \\
\hline C41 & $\mathrm{C}$ & 0.13475 & 0.7318 & 0.4304 \\
\hline C42 & C & -0.09628 & 0.54725 & 0.48415 \\
\hline $\mathrm{C} 43$ & $C$ & -0.12124 & 0.51478 & 0.50942 \\
\hline $\mathrm{C} 44$ & $C$ & -0.1143 & 0.48252 & 0.59957 \\
\hline $\mathrm{C} 45$ & $C$ & -0.08214 & 0.48413 & 0.66549 \\
\hline C46 & C & -0.05806 & 0.51766 & 0.64599 \\
\hline C47 & C & -0.09672 & 0.68664 & 0.3902 \\
\hline $\mathrm{C} 48$ & $C$ & -0.12342 & 0.71688 & 0.38957 \\
\hline C49 & $\mathrm{C}$ & -0.11952 & 0.75352 & 0.4571 \\
\hline C50 & $\mathrm{C}$ & -0.08859 & 0.7593 & 0.52918 \\
\hline C51 & $C$ & -0.0623 & 0.72851 & 0.53356 \\
\hline N52 & $\mathrm{N}$ & 0.23614 & 0.46765 & 0.42774 \\
\hline C53 & $C$ & 0.23391 & 0.42987 & 0.40525 \\
\hline C54 & $C$ & 0.26579 & 0.40312 & 0.42202 \\
\hline C55 & $C$ & 0.30157 & 0.41791 & 0.43984 \\
\hline C56 & $C$ & 0.33133 & 0.39215 & 0.45514 \\
\hline C57 & $C$ & 0.32581 & 0.35114 & 0.45529 \\
\hline C58 & $C$ & 0.28998 & 0.33631 & 0.43835 \\
\hline C59 & $C$ & 0.2604 & 0.36208 & 0.42089 \\
\hline N60 & $\mathrm{N}$ & 0.21711 & 0.80335 & 0.32451 \\
\hline N61 & $\mathrm{N}$ & -0.14874 & 0.78301 & 0.45679 \\
\hline N62 & $\mathrm{N}$ & -0.13824 & 0.44747 & 0.62157 \\
\hline C63 & $C$ & -0.1725 & 0.44463 & 0.59289 \\
\hline C64 & C & -0.1941 & 0.40687 & 0.60823 \\
\hline $\mathrm{C} 65$ & $C$ & -0.18092 & 0.37294 & 0.67133 \\
\hline C66 & $\mathrm{C}$ & 0.31444 & 0.8533 & 0.19037 \\
\hline $\mathrm{C} 67$ & $C$ & 0.27329 & 0.84632 & 0.27681 \\
\hline C68 & C & 0.25516 & 0.87382 & 0.39202 \\
\hline C69 & $C$ & 0.2757 & 0.90668 & 0.42427 \\
\hline $\mathrm{C} 70$ & $C$ & 0.25275 & 0.81055 & 0.24412 \\
\hline C71 & $C$ & -0.20236 & 0.91385 & 0.43937 \\
\hline $\mathrm{C} 72$ & C & -0.17155 & 0.88831 & 0.44441 \\
\hline $\mathrm{C} 73$ & C & -0.17811 & 0.84761 & 0.46679 \\
\hline
\end{tabular}




\begin{tabular}{|c|c|c|c|c|}
\hline $\mathrm{C} 74$ & C & -0.21621 & 0.83298 & 0.4857 \\
\hline C75 & C & -0.24707 & 0.85857 & 0.48307 \\
\hline C76 & C & -0.14513 & 0.82093 & 0.47007 \\
\hline $\mathrm{H} 77$ & $\mathrm{H}$ & 0.07622 & 0.72159 & 0.29513 \\
\hline $\mathrm{H} 78$ & $\mathrm{H}$ & 0.00688 & 0.71886 & 0.34656 \\
\hline $\mathrm{H} 79$ & $\mathrm{H}$ & 0.07998 & 0.52742 & 0.47491 \\
\hline H80 & $\mathrm{H}$ & 0.01239 & 0.52357 & 0.4971 \\
\hline H81 & $\mathrm{H}$ & -0.09084 & 0.62191 & 0.56762 \\
\hline H82 & $\mathrm{H}$ & 0.17723 & 0.6307 & 0.32967 \\
\hline $\mathrm{H} 83$ & $\mathrm{H}$ & 0.13107 & 0.51684 & 0.25868 \\
\hline H84 & $\mathrm{H}$ & 0.17619 & 0.46365 & 0.27733 \\
\hline H85 & $\mathrm{H}$ & 0.23257 & 0.53914 & 0.54844 \\
\hline H86 & $\mathrm{H}$ & 0.18535 & 0.59116 & 0.54259 \\
\hline H87 & $\mathrm{H}$ & 0.19254 & 0.68233 & 0.11816 \\
\hline H88 & $\mathrm{H}$ & 0.23284 & 0.74098 & 0.11041 \\
\hline H89 & $\mathrm{H}$ & 0.14797 & 0.78832 & 0.51398 \\
\hline $\mathrm{H} 90$ & $\mathrm{H}$ & 0.10805 & 0.72903 & 0.52319 \\
\hline H91 & $\mathrm{H}$ & -0.1019 & 0.57062 & 0.40829 \\
\hline H92 & $\mathrm{H}$ & -0.1447 & 0.51474 & 0.45119 \\
\hline H93 & $\mathrm{H}$ & -0.07653 & 0.45985 & 0.73712 \\
\hline H94 & $\mathrm{H}$ & -0.03568 & 0.51911 & 0.71006 \\
\hline H95 & $\mathrm{H}$ & -0.10054 & 0.65876 & 0.33567 \\
\hline H96 & $\mathrm{H}$ & -0.14718 & 0.71183 & 0.33471 \\
\hline H97 & $\mathrm{H}$ & -0.0856 & 0.78679 & 0.58773 \\
\hline H98 & $\mathrm{H}$ & -0.03993 & 0.73291 & 0.59636 \\
\hline H99 & $\mathrm{H}$ & 0.20804 & 0.41717 & 0.38056 \\
\hline $\mathrm{H} 100$ & $\mathrm{H}$ & 0.30671 & 0.44939 & 0.44091 \\
\hline H101 & $\mathrm{H}$ & 0.35869 & 0.4043 & 0.46757 \\
\hline H102 & $\mathrm{H}$ & 0.28501 & 0.30481 & 0.43635 \\
\hline $\mathrm{H} 103$ & $\mathrm{H}$ & 0.23317 & 0.34991 & 0.40701 \\
\hline H104 & $\mathrm{H}$ & -0.18611 & 0.47 & 0.55451 \\
\hline H105 & $\mathrm{H}$ & -0.15471 & 0.37384 & 0.71349 \\
\hline H106 & $\mathrm{H}$ & 0.32942 & 0.83299 & 0.09942 \\
\hline $\mathrm{H} 107$ & $\mathrm{H}$ & 0.22512 & 0.86983 & 0.45878 \\
\hline $\mathrm{H} 108$ & $\mathrm{H}$ & -0.23871 & 0.43114 & 0.5064 \\
\hline $\mathrm{H} 109$ & $\mathrm{H}$ & 0.26857 & 0.79004 & 0.15624 \\
\hline $\mathrm{H} 110$ & $\mathrm{H}$ & -0.1425 & 0.90033 & 0.42978 \\
\hline $\mathrm{H} 111$ & $\mathrm{H}$ & -0.22228 & 0.80179 & 0.50401 \\
\hline $\mathrm{H} 112$ & $\mathrm{H}$ & -0.11748 & 0.83358 & 0.47629 \\
\hline C113 & C & 0.31496 & 0.91318 & 0.34359 \\
\hline C114 & C & 0.33409 & 0.88639 & 0.22636 \\
\hline H115 & $\mathrm{H}$ & 0.36422 & 0.89109 & 0.16209 \\
\hline H116 & $\mathrm{H}$ & 0.2608 & 0.92712 & 0.51506 \\
\hline C117 & C & 0.35803 & 0.32448 & 0.46846 \\
\hline N118 & $\mathrm{N}$ & 0.35152 & 0.28746 & 0.50473 \\
\hline C119 & C & 0.38071 & 0.25847 & 0.5127 \\
\hline C120 & C & 0.41956 & 0.26124 & 0.42047 \\
\hline C121 & C & 0.44582 & 0.23013 & 0.41779 \\
\hline C122 & C & 0.43388 & 0.19568 & 0.50643 \\
\hline C123 & C & 0.39548 & 0.19378 & 0.60303 \\
\hline C124 & C & 0.36919 & 0.22471 & 0.605 \\
\hline C125 & C & 0.45918 & 0.1597 & 0.48311 \\
\hline C126 & C & 0.33514 & 0.94831 & 0.38503 \\
\hline N127 & $\mathrm{N}$ & 0.37208 & 0.95469 & 0.3212 \\
\hline C128 & C & 0.39463 & 0.98776 & 0.3526 \\
\hline C129 & C & 0.38067 & 1.01425 & 0.47852 \\
\hline C130 & C & 0.40338 & 1.04644 & 0.50067 \\
\hline C131 & C & 0.44062 & 1.05302 & 0.3989 \\
\hline C132 & C & 0.4543 & 1.02663 & 0.27316 \\
\hline C133 & C & 0.43194 & 0.99394 & 0.25254 \\
\hline C134 & C & 0.49817 & 0.15914 & 0.49795 \\
\hline C135 & C & 0.52041 & 0.12377 & 0.46699 \\
\hline C136 & C & 0.5029 & 1.08844 & 0.42988 \\
\hline C137 & C & 0.46324 & 1.08892 & 0.42172 \\
\hline C138 & C & 0.44288 & 0.12477 & 0.44335 \\
\hline C139 & C & 0.56007 & 0.12355 & 0.4768 \\
\hline C140 & C & 0.57725 & 0.1586 & 0.51835 \\
\hline C141 & C & 0.55322 & 0.19179 & 0.56402 \\
\hline C142 & C & 0.51499 & 0.19243 & 0.54947 \\
\hline C143 & C & 0.52523 & 1.0536 & 0.40783 \\
\hline C144 & C & 0.5641 & 1.05362 & 0.41362 \\
\hline C145 & C & 0.58224 & 1.08816 & 0.4472 \\
\hline C146 & C & -0.3824 & 0.15896 & 0.51662 \\
\hline C147 & C & -0.36106 & 0.12375 & 0.48202 \\
\hline C148 & C & -0.37801 & 1.08836 & 0.45143 \\
\hline N149 & $\mathrm{N}$ & -0.26818 & 0.96172 & 0.40791 \\
\hline C150 & C & -0.29822 & 0.9905 & 0.40955 \\
\hline
\end{tabular}




\begin{tabular}{|c|c|c|c|c|}
\hline C151 & $C$ & -0.33498 & 0.98917 & 0.52002 \\
\hline C152 & C & -0.36174 & 1.02005 & 0.52845 \\
\hline C153 & C & -0.35255 & 1.05262 & 0.42633 \\
\hline C154 & C & -0.31626 & 1.05331 & 0.31356 \\
\hline C155 & $C$ & -0.28935 & 1.02269 & 0.30618 \\
\hline N156 & $\mathrm{N}$ & -0.28867 & 0.2954 & 0.56826 \\
\hline C157 & C & -0.31215 & 0.26101 & 0.56203 \\
\hline C158 & C & -0.30668 & 0.22471 & 0.63141 \\
\hline C159 & C & -0.33086 & 0.19258 & 0.62328 \\
\hline C160 & C & -0.36028 & 0.19524 & 0.54138 \\
\hline C161 & C & -0.36499 & 0.23144 & 0.46958 \\
\hline C162 & C & -0.34176 & 0.26402 & 0.48281 \\
\hline H163 & $\mathrm{H}$ & -0.24827 & 0.2728 & 0.68102 \\
\hline H164 & $\mathrm{H}$ & -0.30317 & 0.91407 & 0.5007 \\
\hline H165 & $\mathrm{H}$ & 0.38713 & 0.33664 & 0.45072 \\
\hline H166 & $\mathrm{H}$ & 0.42925 & 0.28644 & 0.34478 \\
\hline H167 & $\mathrm{H}$ & 0.47512 & 0.23248 & 0.34273 \\
\hline H168 & $\mathrm{H}$ & 0.38545 & 0.16766 & 0.67308 \\
\hline H169 & $\mathrm{H}$ & 0.3394 & 0.22199 & 0.67626 \\
\hline H170 & $\mathrm{H}$ & 0.35267 & 1.01029 & 0.56213 \\
\hline H171 & $\mathrm{H}$ & 0.39198 & 1.06608 & 0.59945 \\
\hline H172 & $\mathrm{H}$ & 0.4822 & 1.03139 & 0.1902 \\
\hline H173 & $\mathrm{H}$ & 0.4432 & 0.97404 & 0.15458 \\
\hline H174 & $\mathrm{H}$ & 0.41327 & 0.12543 & 0.42975 \\
\hline H175 & $\mathrm{H}$ & 0.56347 & 0.21772 & 0.61293 \\
\hline H176 & $\mathrm{H}$ & 0.4986 & 0.21934 & 0.58109 \\
\hline H177 & $\mathrm{H}$ & 0.51296 & 1.02544 & 0.38951 \\
\hline H178 & $\mathrm{H}$ & 0.57965 & 1.02593 & 0.39241 \\
\hline H179 & $\mathrm{H}$ & -0.33022 & 0.12369 & 0.47813 \\
\hline H180 & $\mathrm{H}$ & -0.3424 & 0.96548 & 0.60527 \\
\hline H181 & $\mathrm{H}$ & -0.38907 & 1.01924 & 0.61862 \\
\hline H182 & $\mathrm{H}$ & -0.30832 & 1.0782 & 0.23408 \\
\hline H183 & $\mathrm{H}$ & -0.26117 & 1.02434 & 0.22113 \\
\hline H184 & $\mathrm{H}$ & -0.28456 & 0.22086 & 0.69525 \\
\hline H185 & $\mathrm{H}$ & -0.3263 & 0.1654 & 0.68102 \\
\hline H186 & $\mathrm{H}$ & -0.38554 & 0.23429 & 0.39736 \\
\hline H187 & $\mathrm{H}$ & -0.346 & 0.29151 & 0.42629 \\
\hline H188 & $\mathrm{H}$ & 0.31798 & 0.96887 & 0.46917 \\
\hline C189 & C & 0.19639 & 0.22572 & 1.12218 \\
\hline C190 & C & 0.16063 & 0.22388 & 1.06433 \\
\hline C191 & C & 0.14828 & 0.25839 & 1.00347 \\
\hline C192 & C & 0.16957 & 0.29334 & 0.99656 \\
\hline C193 & C & 0.13931 & 0.18623 & 1.06933 \\
\hline H194 & $\mathrm{H}$ & 0.20669 & 0.19982 & 1.17172 \\
\hline H195 & $\mathrm{H}$ & 0.12231 & 0.25841 & 0.95957 \\
\hline C196 & C & 0.12421 & 0.81385 & 0.90039 \\
\hline C197 & C & 0.15755 & 0.78738 & 0.89998 \\
\hline H198 & $\mathrm{H}$ & 0.20162 & 0.83328 & 0.8628 \\
\hline H199 & $\mathrm{H}$ & 0.12185 & 0.73474 & 0.94002 \\
\hline C200 & C & 0.46017 & 0.58228 & 0.77982 \\
\hline C201 & C & 0.4197 & 0.58078 & 0.81117 \\
\hline C202 & $\mathrm{C}$ & 0.39908 & 0.5464 & 0.8712 \\
\hline C203 & C & 0.42053 & 0.51147 & 0.88293 \\
\hline C204 & C & 0.46222 & 0.51287 & 0.84759 \\
\hline C205 & C & 0.48192 & 0.54922 & 0.80336 \\
\hline C206 & C & 0.48398 & 0.47816 & 0.8593 \\
\hline C207 & C & 0.46344 & 0.44267 & 0.9003 \\
\hline C208 & $C$ & 0.42313 & 0.44075 & 0.92231 \\
\hline C209 & C & 0.40056 & 0.47498 & 0.92441 \\
\hline C210 & $C$ & 0.35768 & 0.54557 & 0.91566 \\
\hline C211 & C & 0.33881 & 0.51002 & 0.96833 \\
\hline C212 & C & 0.35887 & 0.47418 & 0.96418 \\
\hline C213 & C & 0.52296 & 0.55139 & 0.78006 \\
\hline C214 & $\mathrm{C}$ & 0.54382 & 0.5169 & 0.79026 \\
\hline C215 & C & 0.52545 & 0.48025 & 0.82994 \\
\hline C216 & $C$ & 0.54496 & 0.58942 & 0.76132 \\
\hline C217 & C & 0.5513 & 0.44612 & 0.84566 \\
\hline C218 & C & 0.33218 & 0.58007 & 0.90333 \\
\hline C219 & C & 0.33404 & 0.4375 & 0.99122 \\
\hline C220 & C & 0.54975 & 0.40963 & 0.77028 \\
\hline C221 & C & 0.57606 & 0.37921 & 0.77926 \\
\hline C222 & C & 0.6055 & 0.38528 & 0.859 \\
\hline C223 & C & 0.60799 & 0.42199 & 0.92843 \\
\hline C224 & $C$ & 0.58092 & 0.45169 & 0.92476 \\
\hline C225 & C & 0.58051 & 0.59362 & 0.64541 \\
\hline C226 & C & 0.6037 & 0.6273 & 0.6417 \\
\hline C227 & C & 0.59164 & 0.65747 & 0.75466 \\
\hline
\end{tabular}




\begin{tabular}{|c|c|c|c|c|}
\hline C228 & C & 0.55566 & 0.65374 & 0.86654 \\
\hline C229 & C & 0.53278 & 0.61999 & 0.8712 \\
\hline C230 & C & 0.30175 & 0.43544 & 0.92495 \\
\hline C231 & C & 0.27679 & 0.40297 & 0.95022 \\
\hline C232 & $C$ & 0.28373 & 0.37071 & 1.04038 \\
\hline C233 & C & 0.31589 & 0.37232 & 1.1063 \\
\hline C234 & C & 0.33997 & 0.40585 & 1.08679 \\
\hline C235 & C & 0.30131 & 0.57483 & 0.83101 \\
\hline C236 & C & 0.27461 & 0.60507 & 0.83038 \\
\hline C237 & C & 0.27851 & 0.64171 & 0.8979 \\
\hline C238 & C & 0.30944 & 0.64749 & 0.96999 \\
\hline C239 & C & 0.33573 & 0.6167 & 0.97436 \\
\hline N240 & $\mathrm{N}$ & 0.63417 & 0.35584 & 0.86855 \\
\hline C241 & $C$ & 0.63194 & 0.31806 & 0.84605 \\
\hline C242 & C & 0.66382 & 0.29131 & 0.86283 \\
\hline C243 & C & 0.6996 & 0.3061 & 0.88064 \\
\hline C244 & C & 0.72936 & 0.28034 & 0.89594 \\
\hline C245 & C & 0.72384 & 0.23933 & 0.8961 \\
\hline C246 & C & 0.68801 & 0.2245 & 0.87915 \\
\hline C247 & C & 0.65843 & 0.25027 & 0.8617 \\
\hline N248 & $\mathrm{N}$ & 0.61514 & 0.69154 & 0.76532 \\
\hline N249 & $\mathrm{N}$ & 0.24929 & 0.6712 & 0.89759 \\
\hline N250 & $\mathrm{N}$ & 0.25979 & 0.33566 & 1.06238 \\
\hline C251 & C & 0.22553 & 0.33282 & 1.03369 \\
\hline C252 & C & 0.20393 & 0.29506 & 1.04903 \\
\hline C253 & C & 0.21711 & 0.26113 & 1.11213 \\
\hline C254 & C & 0.71247 & 0.74149 & 0.63117 \\
\hline C255 & C & 0.67132 & 0.73451 & 0.71762 \\
\hline C256 & $C$ & 0.65319 & 0.76201 & 0.83282 \\
\hline C257 & C & 0.67373 & 0.79487 & 0.86507 \\
\hline C258 & C & 0.65078 & 0.69874 & 0.68492 \\
\hline C259 & C & 0.19567 & 0.80204 & 0.88018 \\
\hline C260 & C & 0.22648 & 0.7765 & 0.88522 \\
\hline C261 & C & 0.21992 & 0.7358 & 0.9076 \\
\hline C262 & C & 0.18182 & 0.72117 & 0.9265 \\
\hline C263 & C & 0.15096 & 0.74676 & 0.92388 \\
\hline C264 & C & 0.2529 & 0.70912 & 0.91087 \\
\hline H265 & $\mathrm{H}$ & 0.47425 & 0.60978 & 0.73593 \\
\hline H266 & $\mathrm{H}$ & 0.40491 & 0.60705 & 0.78737 \\
\hline H267 & $\mathrm{H}$ & 0.47801 & 0.41561 & 0.91571 \\
\hline H268 & $\mathrm{H}$ & 0.41042 & 0.41176 & 0.93791 \\
\hline H269 & $\mathrm{H}$ & 0.30719 & 0.5101 & 1.00842 \\
\hline H270 & $\mathrm{H}$ & 0.57526 & 0.51889 & 0.77047 \\
\hline H271 & $\mathrm{H}$ & 0.5291 & 0.40503 & 0.69948 \\
\hline $\mathrm{H} 272$ & $\mathrm{H}$ & 0.57422 & 0.35184 & 0.71814 \\
\hline H273 & $\mathrm{H}$ & 0.6306 & 0.42733 & 0.98924 \\
\hline $\mathrm{H} 274$ & $\mathrm{H}$ & 0.58338 & 0.47935 & 0.98339 \\
\hline H275 & $\mathrm{H}$ & 0.59057 & 0.57052 & 0.55897 \\
\hline H276 & $\mathrm{H}$ & 0.63087 & 0.62917 & 0.55122 \\
\hline H277 & $\mathrm{H}$ & 0.546 & 0.67651 & 0.95479 \\
\hline H278 & $\mathrm{H}$ & 0.50608 & 0.61722 & 0.964 \\
\hline H279 & $\mathrm{H}$ & 0.29613 & 0.45881 & 0.8491 \\
\hline $\mathrm{H} 280$ & $\mathrm{H}$ & 0.25333 & 0.40293 & 0.89199 \\
\hline H281 & $\mathrm{H}$ & 0.3215 & 0.34804 & 1.17793 \\
\hline H282 & $\mathrm{H}$ & 0.36235 & 0.4073 & 1.15086 \\
\hline $\mathrm{H} 283$ & $\mathrm{H}$ & 0.29749 & 0.54695 & 0.77648 \\
\hline $\mathrm{H} 284$ & $\mathrm{H}$ & 0.25085 & 0.60002 & 0.77552 \\
\hline H285 & $\mathrm{H}$ & 0.31243 & 0.67498 & 1.02853 \\
\hline $\mathrm{H} 286$ & $\mathrm{H}$ & 0.3581 & 0.6211 & 1.03717 \\
\hline $\mathrm{H} 287$ & $\mathrm{H}$ & 0.60607 & 0.30536 & 0.82136 \\
\hline $\mathrm{H} 288$ & $\mathrm{H}$ & 0.70474 & 0.33758 & 0.88171 \\
\hline H289 & $\mathrm{H}$ & 0.75672 & 0.29249 & 0.90837 \\
\hline $\mathrm{H} 290$ & $\mathrm{H}$ & 0.68304 & 0.193 & 0.87716 \\
\hline $\mathrm{H} 291$ & $\mathrm{H}$ & 0.6312 & 0.2381 & 0.84781 \\
\hline H292 & $\mathrm{H}$ & 0.21192 & 0.35819 & 0.99532 \\
\hline $\mathrm{H} 293$ & $\mathrm{H}$ & 0.24332 & 0.26203 & 1.1543 \\
\hline $\mathrm{H} 294$ & $\mathrm{H}$ & 0.72745 & 0.72118 & 0.54022 \\
\hline $\mathrm{H} 295$ & $\mathrm{H}$ & 0.62315 & 0.75802 & 0.89959 \\
\hline H296 & $\mathrm{H}$ & 0.15932 & 0.31933 & 0.9472 \\
\hline H297 & $\mathrm{H}$ & 0.6666 & 0.67823 & 0.59704 \\
\hline H298 & $\mathrm{H}$ & 0.25553 & 0.78852 & 0.87059 \\
\hline H299 & $\mathrm{H}$ & 0.17575 & 0.68998 & 0.94481 \\
\hline H300 & $\mathrm{H}$ & 0.28055 & 0.72177 & 0.91709 \\
\hline C301 & C & 0.71299 & 0.80137 & 0.7844 \\
\hline C302 & C & 0.73212 & 0.77458 & 0.66716 \\
\hline H303 & $\mathrm{H}$ & 0.76225 & 0.77928 & 0.6029 \\
\hline H304 & $\mathrm{H}$ & 0.65883 & 0.81531 & 0.95587 \\
\hline
\end{tabular}




\begin{tabular}{|c|c|c|c|c|}
\hline C305 & $C$ & 0.75606 & 0.21267 & 0.90926 \\
\hline N306 & $\mathrm{N}$ & 0.74955 & 0.17565 & 0.94554 \\
\hline C307 & C & 0.77874 & 0.14666 & 0.95351 \\
\hline C308 & C & 0.81759 & 0.14943 & 0.86127 \\
\hline C309 & $C$ & 0.84385 & 0.11832 & 0.8586 \\
\hline C310 & C & 0.83191 & 0.08387 & 0.94723 \\
\hline C311 & C & 0.79351 & 0.08197 & 1.04384 \\
\hline C312 & C & 0.76722 & 0.1129 & 1.04581 \\
\hline C313 & $\mathrm{C}$ & 0.85721 & 0.04789 & 0.92392 \\
\hline C314 & C & 0.73317 & 0.8365 & 0.82583 \\
\hline N315 & $\mathrm{N}$ & 0.77011 & 0.84288 & 0.762 \\
\hline C316 & C & 0.79266 & 0.87595 & 0.7934 \\
\hline C317 & $\mathrm{C}$ & 0.7787 & 0.90244 & 0.91933 \\
\hline C318 & C & 0.80141 & 0.93463 & 0.94148 \\
\hline C319 & C & 0.83865 & 0.94121 & 0.83971 \\
\hline C320 & C & 0.85233 & 0.91482 & 0.71396 \\
\hline C321 & $\mathrm{C}$ & 0.82997 & 0.88213 & 0.69335 \\
\hline C322 & C & 0.8962 & 0.04733 & 0.93876 \\
\hline C323 & C & 0.91844 & 0.01196 & 0.90779 \\
\hline C324 & C & 0.90093 & 0.97663 & 0.87068 \\
\hline C325 & C & 0.86127 & 0.97711 & 0.86252 \\
\hline C326 & C & 0.84091 & 0.01296 & 0.88416 \\
\hline C327 & C & 0.9581 & 0.01174 & 0.91761 \\
\hline C328 & C & 0.97528 & 0.04679 & 0.95915 \\
\hline C329 & $C$ & 0.95125 & 0.07998 & 1.00482 \\
\hline C330 & C & 0.91302 & 0.08062 & 0.99027 \\
\hline C331 & C & 0.92326 & 0.94179 & 0.84864 \\
\hline C332 & C & 0.96213 & 0.94181 & 0.85443 \\
\hline C333 & C & 0.98027 & 0.97635 & 0.88801 \\
\hline C334 & C & 0.01563 & 0.04715 & 0.95742 \\
\hline C335 & C & 0.03697 & 0.01194 & 0.92283 \\
\hline C336 & C & 0.02002 & 0.97655 & 0.89224 \\
\hline N337 & $\mathrm{N}$ & 0.12985 & 0.84991 & 0.84871 \\
\hline C338 & C & 0.09981 & 0.87869 & 0.85036 \\
\hline C339 & C & 0.06305 & 0.87736 & 0.96082 \\
\hline C340 & C & 0.03629 & 0.90824 & 0.96926 \\
\hline C341 & C & 0.04548 & 0.94081 & 0.86713 \\
\hline C342 & C & 0.08177 & 0.9415 & 0.75436 \\
\hline C343 & C & 0.10868 & 0.91088 & 0.74698 \\
\hline N344 & $\mathrm{N}$ & 0.10936 & 0.18359 & 1.00906 \\
\hline C345 & C & 0.08588 & 0.1492 & 1.00284 \\
\hline C346 & C & 0.09135 & 0.1129 & 1.07222 \\
\hline C347 & C & 0.06717 & 0.08077 & 1.06409 \\
\hline C348 & C & 0.03775 & 0.08343 & 0.98219 \\
\hline C349 & C & 0.03304 & 0.11963 & 0.91038 \\
\hline C350 & C & 0.05627 & 0.15221 & 0.92361 \\
\hline H351 & $\mathrm{H}$ & 0.14976 & 0.16099 & 1.12183 \\
\hline H352 & $\mathrm{H}$ & 0.09486 & 0.80226 & 0.94151 \\
\hline H353 & $\mathrm{H}$ & 0.78516 & 0.22483 & 0.89152 \\
\hline H354 & $\mathrm{H}$ & 0.82728 & 0.17463 & 0.78559 \\
\hline H355 & $\mathrm{H}$ & 0.87315 & 0.12067 & 0.78354 \\
\hline H356 & $\mathrm{H}$ & 0.78348 & 0.05585 & 1.11388 \\
\hline H357 & $\mathrm{H}$ & 0.73743 & 0.11018 & 1.11706 \\
\hline H358 & $\mathrm{H}$ & 0.7507 & 0.89848 & 1.00293 \\
\hline H359 & $\mathrm{H}$ & 0.79001 & 0.95427 & 1.04026 \\
\hline H360 & $\mathrm{H}$ & 0.88023 & 0.91958 & 0.631 \\
\hline H361 & $\mathrm{H}$ & 0.84123 & 0.86223 & 0.59538 \\
\hline H362 & $\mathrm{H}$ & 0.8113 & 0.01362 & 0.87056 \\
\hline H363 & $\mathrm{H}$ & 0.9615 & 0.10591 & 1.05374 \\
\hline H364 & $\mathrm{H}$ & 0.89663 & 0.10753 & 1.02189 \\
\hline H365 & $\mathrm{H}$ & 0.91099 & 0.91363 & 0.83032 \\
\hline H366 & $\mathrm{H}$ & 0.97768 & 0.91412 & 0.83322 \\
\hline H367 & $\mathrm{H}$ & 0.06781 & 0.01188 & 0.91894 \\
\hline H368 & $\mathrm{H}$ & 0.05563 & 0.85367 & 1.04607 \\
\hline H369 & $\mathrm{H}$ & 0.00896 & 0.90743 & 1.05943 \\
\hline H370 & $\mathrm{H}$ & 0.08971 & 0.96639 & 0.67488 \\
\hline H371 & $\mathrm{H}$ & 0.13686 & 0.91253 & 0.66193 \\
\hline H372 & $\mathrm{H}$ & 0.11347 & 0.10905 & 1.13606 \\
\hline H373 & $\mathrm{H}$ & 0.07173 & 0.05359 & 1.12182 \\
\hline H374 & $\mathrm{H}$ & 0.01249 & 0.12248 & 0.83816 \\
\hline H375 & $\mathrm{H}$ & 0.05203 & 0.1797 & 0.8671 \\
\hline H376 & $\mathrm{H}$ & 0.71601 & 0.85706 & 0.90998 \\
\hline
\end{tabular}


Table S4. Detailed atom information of the simulated TAPT-BTCA COF in quasi-AB stacking mode. $\mathrm{a}=18.7121, \mathrm{~b}=18.7121, \mathrm{c}=6.8832 ;$ alpha $=$ beta $=90^{\circ}$, gamma $=120.0^{\circ} ; \mathrm{P}_{1}$ space group

\begin{tabular}{|c|c|c|c|c|}
\hline & & $x$ & $Y$ & Z \\
\hline C1 & C & 0.95049 & -0.07593 & -0.0262 \\
\hline $\mathrm{N} 2$ & $\mathrm{~N}$ & 1.03419 & -0.03042 & -0.0262 \\
\hline $\mathrm{C} 3$ & $C$ & 0.89498 & 0.08917 & -0.0262 \\
\hline $\mathrm{C} 4$ & $\mathrm{C}$ & 0.93421 & 0.17578 & -0.0262 \\
\hline $\mathrm{C} 5$ & $\mathrm{C}$ & 0.88794 & 0.21567 & -0.0262 \\
\hline C6 & C & 0.80106 & 0.16944 & -0.0262 \\
\hline $\mathrm{C} 7$ & $\mathrm{C}$ & 0.76212 & 0.08325 & -0.0262 \\
\hline $\mathrm{C} 8$ & $\mathrm{C}$ & 0.80831 & 0.04333 & -0.0262 \\
\hline N9 & $\mathrm{N}$ & 0.74974 & 0.20649 & -0.0262 \\
\hline C10 & $\mathrm{C}$ & 0.77379 & 0.28477 & -0.0262 \\
\hline C11 & $\mathrm{C}$ & 0.71293 & 0.31307 & -0.0262 \\
\hline $\mathrm{C} 12$ & $\mathrm{C}$ & 0.62769 & 0.25668 & -0.0262 \\
\hline $\mathrm{H} 13$ & $\mathrm{H}$ & 1.00086 & 0.21303 & -0.0262 \\
\hline $\mathrm{H} 14$ & $\mathrm{H}$ & 0.92106 & 0.28224 & -0.0262 \\
\hline $\mathrm{H} 15$ & $\mathrm{H}$ & 0.6954 & 0.04676 & -0.0262 \\
\hline $\mathrm{H} 16$ & $\mathrm{H}$ & 0.77595 & -0.02346 & -0.0262 \\
\hline $\mathrm{H} 17$ & $\mathrm{H}$ & 0.83849 & 0.33088 & -0.0262 \\
\hline $\mathrm{H} 18$ & $\mathrm{H}$ & 0.60543 & 0.19095 & -0.0262 \\
\hline C19 & $\mathrm{C}$ & 1.07338 & 0.05333 & -0.0262 \\
\hline $\mathrm{N} 20$ & $\mathrm{~N}$ & 1.02787 & 0.09152 & -0.0262 \\
\hline $\mathrm{C} 21$ & $\mathrm{C}$ & 0.90828 & -0.16727 & -0.0262 \\
\hline $\mathrm{C} 22$ & $\mathrm{C}$ & 0.82167 & -0.21466 & -0.0262 \\
\hline $\mathrm{C} 23$ & C & 0.78177 & -0.30082 & -0.0262 \\
\hline $\mathrm{C} 24$ & $\mathrm{C}$ & 0.828 & 0.65853 & -0.0262 \\
\hline $\mathrm{C} 25$ & $\mathrm{C}$ & 0.9142 & -0.29421 & -0.0262 \\
\hline $\mathrm{C} 26$ & C & 0.95412 & -0.20811 & -0.0262 \\
\hline N27 & $\mathrm{N}$ & 0.79096 & 0.57016 & -0.0262 \\
\hline $\mathrm{C} 28$ & $\mathrm{C}$ & 0.71268 & 0.51593 & -0.0262 \\
\hline $\mathrm{C} 29$ & $C$ & 0.68438 & 0.42678 & -0.0262 \\
\hline C30 & $\mathrm{C}$ & 0.74077 & 0.39792 & -0.0262 \\
\hline H31 & $\mathrm{H}$ & 0.78442 & -0.18525 & -0.0262 \\
\hline $\mathrm{H} 32$ & $\mathrm{H}$ & 0.71521 & 0.66573 & -0.0262 \\
\hline H33 & $\mathrm{H}$ & 0.95068 & -0.32445 & -0.0262 \\
\hline $\mathrm{H} 34$ & $\mathrm{H}$ & 1.02091 & -0.17368 & -0.0262 \\
\hline H35 & $\mathrm{H}$ & 0.66657 & 0.53453 & -0.0262 \\
\hline H36 & $\mathrm{H}$ & 0.8065 & 0.4414 & -0.0262 \\
\hline C37 & $\mathrm{C}$ & 0.94412 & 0.04696 & -0.0262 \\
\hline N38 & $\mathrm{N}$ & 0.90592 & -0.03674 & -0.0262 \\
\hline C39 & $\mathrm{C}$ & 1.16472 & 0.10247 & -0.0262 \\
\hline $\mathrm{C} 40$ & $\mathrm{C}$ & 1.21211 & 0.06324 & -0.0262 \\
\hline C41 & $\mathrm{C}$ & 1.29827 & 0.10951 & -0.0262 \\
\hline $\mathrm{C} 42$ & $\mathrm{C}$ & 0.33892 & 0.19639 & -0.0262 \\
\hline $\mathrm{C} 43$ & $\mathrm{C}$ & 1.29166 & 0.23532 & -0.0262 \\
\hline $\mathrm{C} 44$ & $\mathrm{C}$ & 1.20556 & 0.18914 & -0.0262 \\
\hline N45 & $\mathrm{N}$ & 0.42728 & 0.24771 & -0.0262 \\
\hline $\mathrm{C} 46$ & $\mathrm{C}$ & 0.48152 & 0.22366 & -0.0262 \\
\hline $\mathrm{C} 47$ & $\mathrm{C}$ & 0.57067 & 0.28452 & -0.0262 \\
\hline $\mathrm{C} 48$ & $\mathrm{C}$ & 0.59953 & 0.36976 & -0.0262 \\
\hline $\mathrm{H} 49$ & $\mathrm{H}$ & 1.1827 & -0.00342 & -0.0262 \\
\hline $\mathrm{H} 50$ & $\mathrm{H}$ & 0.33172 & 0.07639 & -0.0262 \\
\hline H51 & $\mathrm{H}$ & 1.3219 & 0.30205 & -0.0262 \\
\hline H52 & $\mathrm{H}$ & 1.17113 & 0.2215 & -0.0262 \\
\hline H53 & $\mathrm{H}$ & 0.46292 & 0.15896 & -0.0262 \\
\hline H54 & $\mathrm{H}$ & 0.55605 & 0.39201 & -0.0262 \\
\hline C55 & $\mathrm{C}$ & 0.62783 & 0.24928 & 0.5 \\
\hline N56 & $\mathrm{N}$ & 0.71153 & 0.29479 & 0.5 \\
\hline C57 & $\mathrm{C}$ & 0.57232 & 0.41438 & 0.5 \\
\hline C58 & $\mathrm{C}$ & 0.61155 & 0.50099 & 0.5 \\
\hline C59 & $\mathrm{C}$ & 0.56528 & 0.54089 & 0.5 \\
\hline $\mathrm{C} 60$ & $\mathrm{C}$ & 0.4784 & 0.49466 & 0.5 \\
\hline C61 & $\mathrm{C}$ & 0.43946 & 0.40846 & 0.5 \\
\hline C62 & $\mathrm{C}$ & 0.48565 & 0.36855 & 0.5 \\
\hline N63 & $\mathrm{N}$ & 0.42708 & 0.5317 & 0.5 \\
\hline C64 & $\mathrm{C}$ & 0.45112 & 0.60998 & 0.5 \\
\hline C65 & $\mathrm{C}$ & 0.39027 & 0.63828 & 0.5 \\
\hline C66 & $\mathrm{C}$ & 0.30502 & 0.58189 & 0.5 \\
\hline $\mathrm{H} 67$ & $\mathrm{H}$ & 0.6782 & 0.53824 & 0.5 \\
\hline $\mathrm{H} 68$ & $\mathrm{H}$ & 0.5984 & 0.60745 & 0.5 \\
\hline $\mathrm{H} 69$ & $\mathrm{H}$ & 0.37274 & 0.37198 & 0.5 \\
\hline $\mathrm{H} 70$ & $\mathrm{H}$ & 0.45328 & 0.30175 & 0.5 \\
\hline $\mathrm{H} 71$ & $\mathrm{H}$ & 0.51583 & 0.65609 & 0.5 \\
\hline $\mathrm{H} 72$ & $\mathrm{H}$ & 0.28277 & 0.51616 & 0.5 \\
\hline $\mathrm{C} 73$ & $\mathrm{C}$ & 0.75072 & 0.37855 & 0.5 \\
\hline
\end{tabular}




\begin{tabular}{|c|c|c|c|c|}
\hline N74 & $\mathrm{N}$ & 0.70521 & 0.41674 & 0.5 \\
\hline C75 & C & 0.58562 & 0.15794 & 0.5 \\
\hline C76 & C & 0.49901 & 0.11055 & 0.5 \\
\hline C77 & C & 0.45911 & 0.02439 & 0.5 \\
\hline C78 & C & 0.50534 & 0.98374 & 0.5 \\
\hline C79 & C & 0.59154 & 0.031 & 0.5 \\
\hline C80 & C & 0.63145 & 0.1171 & 0.5 \\
\hline N81 & $\mathrm{N}$ & 0.4683 & 0.89538 & 0.5 \\
\hline C82 & C & 0.39002 & 0.84114 & 0.5 \\
\hline C83 & C & 0.36172 & 0.75199 & 0.5 \\
\hline C84 & C & 0.41811 & 0.72314 & 0.5 \\
\hline H85 & $\mathrm{H}$ & 0.46176 & 0.13996 & 0.5 \\
\hline H86 & $\mathrm{H}$ & 0.39255 & 0.99094 & 0.5 \\
\hline H87 & $\mathrm{H}$ & 0.62802 & 0.00076 & 0.5 \\
\hline H88 & $\mathrm{H}$ & 0.69825 & 0.15153 & 0.5 \\
\hline H89 & $\mathrm{H}$ & 0.34391 & 0.85974 & 0.5 \\
\hline H9O & $\mathrm{H}$ & 0.48384 & 0.76661 & 0.5 \\
\hline C91 & C & 0.62145 & 0.37217 & 0.5 \\
\hline N92 & $\mathrm{N}$ & 0.58326 & 0.28847 & 0.5 \\
\hline C93 & C & 0.84206 & 0.42768 & 0.5 \\
\hline C94 & C & 0.88945 & 0.38845 & 0.5 \\
\hline C95 & C & 0.97561 & 0.43472 & 0.5 \\
\hline C96 & C & 0.01626 & 0.5216 & 0.5 \\
\hline C97 & C & 0.969 & 0.56054 & 0.5 \\
\hline C98 & C & 0.8829 & 0.51435 & 0.5 \\
\hline N99 & $\mathrm{N}$ & 0.10462 & 0.57292 & 0.5 \\
\hline C100 & C & 0.15886 & 0.54888 & 0.5 \\
\hline C101 & C & 0.24801 & 0.60973 & 0.5 \\
\hline C102 & C & 0.27686 & 0.69498 & 0.5 \\
\hline H103 & $\mathrm{H}$ & 0.86004 & 0.3218 & 0.5 \\
\hline H104 & $\mathrm{H}$ & 0.00906 & 0.4016 & 0.5 \\
\hline H105 & $\mathrm{H}$ & 0.99924 & 0.62726 & 0.5 \\
\hline H106 & $\mathrm{H}$ & 0.84847 & 0.54672 & 0.5 \\
\hline H107 & $\mathrm{H}$ & 0.14026 & 0.48417 & 0.5 \\
\hline H108 & $\mathrm{H}$ & 0.23339 & 0.71723 & 0.5 \\
\hline
\end{tabular}




\section{References}

(1) Smith, B. J.; Overholts, A. C.; Hwang, N.; Dichtel, W. R. Insight into the Crystallization of Amorphous Imine-Linked Polymer Networks to 2D Covalent Organic Frameworks. Chem. Commun. 2016, 52 (18), 3690-3693.

(2) Dong, J.; Wang, Y.; Liu, G.; Cheng, Y.; Zhao, D. Isoreticular Covalent Organic Frameworks for Hydrocarbon Uptake and Separation: The Important Role of Monomer Planarity. CrystEngComm 2017, 19 (33), 4899-4904.

(3) Xu, H.; Gao, J.; Jiang, D. Stable, Crystalline, Porous, Covalent Organic Frameworks as a Platform for Chiral Organocatalysts. Nat. Chem. 2015, 7 (11), 905-912.

(4) Auras, F.; Ascherl, L.; Hakimioun, A. H.; Margraf, J. T.; Hanusch, F. C.; Reuter, S.; Bessinger, D.; Döblinger, M.; Hettstedt, C.; Karaghiosoff, K.; Herbert, S.; Knochel, P.; Clark, T.; Bein, T. Synchronized Offset Stacking: A Concept for Growing Large-Domain and Highly Crystalline 2D Covalent Organic Frameworks. J. Am. Chem. Soc. 2016, 138 (51), 16703-16710.

(5) Sun, Q.; Fu, C.-W.; Aguila, B.; Perman, J.; Wang, S.; Huang, H.-Y.; Xiao, F.-S.; Ma, S. Pore Environment Control and Enhanced Performance of Enzymes Infiltrated in Covalent Organic Frameworks. J. Am. Chem. Soc. 2018, 140 (3), 984-992.

(6) Zhang, S.; Zheng, Y.; An, H.; Aguila, B.; Yang, C.-X.; Dong, Y.; Xie, W.; Cheng, P.; Zhang, Z.; Chen, Y.; Ma, S. Covalent Organic Frameworks with Chirality Enriched by Biomolecules for Efficient Chiral Separation. Angew. Chem. Int. Ed. 2018, 57 (51), 16754-16759. 